\title{
A decision tool based on bilevel optimization for the allocation of water resources in a hierarchical system
}

\author{
Herminia I. Calvete ${ }^{a} *$ (D) Carmen Galéb $^{b}$, José A. Iranzo ${ }^{c}$ (D) and Pedro M. Mateo ${ }^{d}$ \\ a Dpto. de Métodos Estadísticos, IUMA, Universidad de Zaragoza, Pedro Cerbuna 12, Zaragoza 50009, Spain \\ ${ }^{\mathrm{b}}$ Dpto. de Métodos Estadísticos, IUMA, Universidad de Zaragoza, María de Luna 3, Zaragoza 50018, Spain \\ ${ }^{\mathrm{c}}$ Dpto. de Métodos Estadísticos, IUMA, Universidad de Zaragoza, Atarazana 2, Teruel 44003, Spain \\ ${ }^{\mathrm{d}}$ Dpto. de Métodos Estadísticos, BIFI, Universidad de Zaragoza, Pedro Cerbuna 12, Zaragoza 50009, Spain \\ E-mail: herminia@unizar.es [Calvete]; cgale@unizar.es [Galé]; joseani@unizar.es [Iranzo]; mateo@unizar.es [Mateo]
}

Received 14 April 2020; received in revised form 19 January 2021; accepted 19 January 2021

\begin{abstract}
This paper addresses the optimal allocation of water among competing stakeholders during a finite planning horizon. We focus on those water systems where there are two levels of decision making organized according to a hierarchical framework. At the upper level, a central authority allocates water to demand points having regard to environmental and sustainability issues as well as balancing water users' supply/demand. At the lower level of the hierarchy, demand point managers allocate water to users prioritizing economic strategies. On the other hand, when it comes to allocating limited resources that affect public welfare, the authority in charge can also use different political instruments such as fees to influence the decisions made at those levels of decision making that are not directly within its competence. We propose a multiobjective multifollower bilevel optimization problem that aims to fulfill the central authority goals while including the reaction of the demand point managers in terms of optimization problems as constraints. Using the wellknown Karush-Kuhn-Tucker approach, we transform the bilevel model into an equivalent multiobjective mixed-integer single-level model for which we provide tight big- $M$ values. For the purpose of showing the versatility of the model, extensive computational experiments on a set of instances have been carried out. The results show that the optimization problem can be solved to optimality in small computing times using off-the-shelf mixed-integer solvers even for complex water systems and long planning periods. In addition, they illustrate the effect of imposing fees on the achievement of the central authority's objectives.
\end{abstract}

Keywords: water system management; bilevel optimization; multiobjective; multifollower; exact solution; decision tool

\section{Introduction}

Distributing water has become a very important issue due to its increasing uses and needs. In cases where the demand for water is small compared to its availability, conflicts do not arise. However, in

*Corresponding author.

(C) 2021 The Authors.

International Transactions in Operational Research (C) 2021 International Federation of Operational Research Societies Published by John Wiley \& Sons Ltd, 9600 Garsington Road, Oxford OX4 2DQ, UK and 350 Main St, Malden, MA02148, USA. 
general, water resources are too limited to meet all the needs of water users, and the use of water by one user affects the availability for other users. Moreover, water users usually pursue different and even conflicting objectives. Therefore, water system managers face conflicts when users compete for water and making decisions become increasingly complex. On the other hand, there is an increasing interest in improving water resources management by taking into account sustainability and environmental protection of aquatic ecosystems as well as giving users the opportunity of participating in the decision-making process (Hassing et al., 2009; Ruiz-Villaverde and García-Rubio, 2017). Optimization techniques have provided support to deal with water system management. In the literature, the long-established modeling approach assumes centralized planning, that is, the water distribution decision process is controlled by a single decision maker. In some cases, multiple decision makers are allowed, who agree to collaborate to achieve common goals in a decentralized coordinated planning process. Therefore, optimization models with a single decision level and one or several criteria have been proposed in the literature; see Brown et al. (2015), Calvete and Mateo (1995), Llopis-Albert et al. (2018), Mala-Jetmarova et al. (2017), Roozbahani et al. (2013), Tayfur (2017), Udías et al. (2012), and references therein. Most of these papers, when explicitly taking into account environmental issues besides economic issues, propose a multiobjective model.

However, there are many practical problems in which the agents involved in water allocation are not necessarily willing to cooperate. For some of them, environmental issues must be considered a priority. For others, obtaining the best economic performance is the only thing that matters. In order to deal with this conflict, and bearing in mind that usually a hierarchy can be recognized in the decision-making process with a central authority having a prevailing role, a bilevel optimization approach for the optimal allocation of water is more appropriate, such as that proposed in this paper. This approach allows the central authority the achievement of environmental goals and the use of fees to encourage a balanced allocation of water, while the demand points decide on this allocation. The idea of a central authority influencing decision makers has already been applied in different fields. Among others, Önal et al. (1995) model the distribution of agricultural credits between farm groups aiming to improve the agriculture sector's performance. Amouzegar and Moshirvaziri (1999) deal with managing hazardous waste aiming to maximize social welfare via taxation. Zhao et al. (2013) study how to control water pollution to attain the desired water quality at the lowest environmental cost. Bostian et al. (2015) aim to control nitrogen loading in the watershed to achieve a trade-off between agricultural production and water quality. The review by Sinha et al. (2018) includes several references that deal with the application of bilevel optimization in environmental economics, where an authority or regulator at the upper level of decision making tax those entities at the lower level that are polluting the environment as a result of their operations. In a hierarchized sustainable supply chain network design, Chalmardi and CamachoVallejo (2019) propose a bilevel model in which the government acts as the leader to incentivize the use of cleaner technologies by offering financial incentives to the supply chain's managers. For eco-industrial parks, Aviso et al. (2010) propose a bilevel fuzzy optimization model to explore the effect of charging fees for the purchase of freshwater and for the treatment of wastewater in optimizing the water exchange network of plants and Bi et al. (2019) model the distribution of water to minimize overall water consumption.

Bilevel optimization models those problems having a hierarchical framework in which there are two decision levels whose decision makers, besides having different goals, only have control over some of the decisions to be made. The decision maker at the upper level of the hierarchy aims to 
optimize his/her objective function under a set of constraints that take into account the reaction of the decision maker at the lower level of the hierarchy to the plan of the leader. Therefore, bilevel programs are formulated as optimization problems, which involve another optimization problem in the constraint set. Regarding the application of bilevel optimization in water-allocation problems, Guo et al. (2012) propose a bilevel model for a multireservoir operation model in an interbasin water transfer supply project. The upper level controls the distribution of water resources among water exporting and importing regions using a set of water-transfer rules. The individual reservoir manager, at the lower level of the hierarchy, controls the water-supply process by hedging rules. The authors develop an improved particle swarm optimization that is used to solve both upper and lower level models transferring the value of the corresponding variables from one model to another, and apply it to a three-reservoir system in a province of Northeast China. Zhu et al. (2017) consider the problem of transferring and supplying water and present a bilevel model, which is solved using an adaptive genetic algorithm. The upper level's goal is to minimize the actual annual average transferred water while the lower level aims to minimize water shortage for all users. A fuzzy approach is often taken to solve the bilevel problem when the upper and lower decision makers are able to cooperate with each other. Under this hypothesis, the bilevel problem can usually be solved sequentially and thus it is simpler to handle. This approach is adopted by $\mathrm{Lv}$ et al. (2010) and Xu et al. (2012) for planning water resources management systems. The aim is to balance the degree of satisfaction between the upper and lower levels. Chen et al. (2017) propose a bilevel model in which the upper level decision maker aims to minimize the discharge of pollutants when deciding the water-allocation strategy, whereas the lower level representing the regional authority maximizes the economic benefits. To solve the problem, an interactive algorithm is developed based on the concepts of satisfaction and tolerance membership functions of the fuzzy theory.

The contribution of this paper is to propose a model for water resource allocation in a general complex noncooperative hierarchical water system. The model is flexible enough to allow consideration of very different water system configurations, the evolution over time of the system, and environmental and economic issues. In addition, the model enables the use of fees that can be charged by the decision maker at the highest level of the hierarchy to influence the decisions made at those levels of decision making that are not directly within its competence. The result is a decision tool that can be used to evaluate different strategies in the optimal allocation of the available water when the reaction of the users is included in the decision process.

The study considers the management during a finite planning horizon, divided into time periods, of a water resources system consisting of rivers, reservoirs, distribution channels, and water demand points, each having several water users. Due to water shortages, conflicts arise among the demand points. The aim is to determine how to allocate the available water taking into account the hierarchical structure of the decision process. On the one hand, a central authority representing the government or water system authorities decides on the global amount of water allocated to each demand point. It aims to distribute water in accordance with environmental aspects and the overall satisfaction of user demand, having the possibility of charging fees on water allocated to users. On the other hand, managers at the demand points (which represent water users' communities) decide on the different uses of the allocated water, that is, on the distribution to their water users based on maximizing the net economic return. Both decision levels act under a hierarchical structure. This means that the central authority at the upper level of the decision process has control of the water 
distribution to the demand points, but the reaction of the managers who decide on water uses is taken into account in the model as a constraint.

This issue is modeled as a multiobjective lexicographic linear bilevel optimization problem with a leader and several followers, one for each demand point. First, taking into account that each follower problem only involves its own variables and the variables controlled by the upper level, this model is transformed into a multiobjective lexicographic linear bilevel problem with a single follower. Then, to solve the problem, we propose a procedure that takes advantage of the fact that, after the above-mentioned transformation, the lower level problem is a linear one. Hence, KarushKuhn-Tucker (KKT) conditions are necessary and sufficient for optimality, and the multiobjective lexicographic bilevel problem can be transformed into an equivalent multiobjective lexicographic mixed-integer linear single-level problem by introducing additional binary variables. This linearization process involves the use of big- $M$ constants for which we provide tight valid values. The resulting optimization problem can be solved to optimality using off-the-shelf mixed-integer solvers. It is worth pointing out that the model can efficiently handle complex water systems with long planning periods. To illustrate the performance of this approach, we have generated a set of instances that handle a variety of water system configurations under different water availability scenarios. Extensive computational experiments on this set show the versatility of the model as well as the short computing times required to solve it. As a result, this work provides a mathematical model that can be used as a decision tool by the central authority to assess the degree of fulfillment of its objectives and to quickly evaluate the consequences of different simulated scenarios when deciding water allocation.

The rest of the paper is structured as follows. Following the Introduction section, Section 2 provides a brief summary of bilevel optimization. Section 3 describes in detail the formulation of the bilevel model and the procedure used to solve it. Section 4 presents the results of the computational experiments carried out. Finally, Section 5 concludes the paper.

\section{Background on bilevel optimization problems}

As mentioned above, bilevel optimization has been proposed to deal with hierarchical decision processes with two levels of decision. When there is a leader and a single follower, the bilevel optimization model can be formulated as

$$
\begin{aligned}
& \min _{x} \quad F(x, y) \\
& \text { subject to } \\
& G_{j}(x, y) \leq 0, j=1, \ldots, q, \\
& \text { where, for every } x \text { fixed, } y \text { solves } \\
& \min _{y} \quad f(x, y) \\
& \text { subject to } \\
& g_{h}(x, y) \leq 0, h=1, \ldots, p,
\end{aligned}
$$

where $x \in \mathbb{R}^{n}$ are the upper level variables controlled by the leader, and $y \in \mathbb{R}^{m}$ are the lower level variables controlled by the follower; $F, f: \mathbb{R}^{n+m} \longrightarrow \mathbb{R}$ are the upper and lower level objective 
functions, respectively; and $G_{j}(x, y) \leq 0, g_{h}(x, y) \leq 0$ refer, respectively, to constraints of the upper and lower levels.

The bilevel problem is the leader's problem, who must anticipate the follower's reaction when looking for his/her best decision. The follower is free to optimize his/her objective function once the leader sets the value of the upper level variables. Therefore, for a given $x$, the follower solves the lower level problem:

$$
\begin{array}{ll}
\min _{y} & f(x, y) \\
\text { subject to } & \\
& g_{h}(x, y) \leq 0, \quad h=1, \ldots, p .
\end{array}
$$

Let $V=\left\{(x, y): G_{j}(x, y) \leq 0, j=1, \ldots, q, g_{h}(x, y) \leq 0, h=1, \ldots, p\right\}$. Let $M(x)$ be the optimal solution set of problem (2). Let $T=\{(x, y):(x, y) \in V, y \in M(x)\}$. A point $(x, y) \in T$ is a so-called bilevel feasible solution, that is, a feasible solution of problem (1). A point $x \in \mathbb{R}^{n}$ is called permissible if a point $y \in \mathbb{R}^{m}$ exists so that $(x, y) \in T$. Hence, the bilevel problem (1) can be equivalently formulated as

$$
\begin{array}{ll}
\min _{x} & F(x, y) \\
\text { s.t. } & \\
& (x, y) \in T .
\end{array}
$$

The bilevel problem is nonconvex and difficult to deal with and solve. In fact, complications arise when there are multiple optima in the lower level problem, that is to say, $M(x)$ is not a singleton for some permissible $x$. If the upper level objective function is sensitive to different values of $y \in M(x)$, it is necessary to give a rule to select $y^{*} \in M(x)$ in order to evaluate $F$. Several assumptions have been proposed in the literature, the most common being the optimistic approach, which assumes that the upper level decision maker has the right to influence the lower level decision maker so that the latter selects $y^{*}$ to provide the best value of $F$. In this case, the upper level objective function is minimized over $x$ and $y$. This is the approach taken in this paper. It is also worth noting that even when all the functions involved are linear, the bilevel problem is strongly NP-hard. Calvete et al. (2012) provide an overall view of the main difficulties that arise when dealing with bilevel problems and distinguish them from classical single-level optimization problems. Bard (1998) and Dempe (2002) are textbooks that deal with most of the theoretical issues on bilevel optimization. Colson et al. (2007), Dempe (2018), Sinha et al. (2018), and Dempe and Zemkoho (2020) provide recent surveys that cover applications as well as major theoretical developments.

\section{A multiobjective multifollower bilevel optimization model for water system management}

In this section, we mathematically describe the problem addressed by the central authority when allocating water and formulate the bilevel problem that models it. Water enters the system at various locations and is distributed to demand points or stored in reservoirs; otherwise, it leaves the system. From the demand points, the water is allocated to water users aiming to satisfy their demands. To take into account that demand and inflows change over time, we consider the evolution of the water 
system during a finite planning horizon divided into periods. For the water system management, it is necessary to know not only if there is enough water overall to satisfy demand but if water is available for allocation during the required period. We assume that in each period of the planning horizon, the inflows to the system and every water user demand are known or can be properly estimated. Moreover, minimum downstream requirements and minimum storage in reservoirs are also known and must be prioritized when determining the optimal water distribution to meet environmental and sustainability issues. The goal is to determine the best way of allocating available water. The central authority, playing the role of leader, controls the water that is allocated to the demand points, as well as minimum needs and the flows leaving the system. Each demand point, acting as a follower, controls the allocation of water to its water users.

\subsection{Assumptions, notations, and variables}

Let $T$ denote the set of periods of the planning horizon. Let $R$ be the set of reservoirs, $W$ be the set of demand points, and $K$ be the set of water users. Each reservoir $r \in R$ is connected to other reservoirs and/or to demand points. Let $R_{r} \subseteq R$ and $W_{r} \subseteq W$ be, respectively, the sets of reservoirs and demand points to which $r$ is connected. This means that water can flow from the reservoir $r$ to any node in $R_{r} \cup W_{r}$. Each demand point $w \in W$ is only connected to the set $K_{w} \subseteq K$ of water users to which $w$ can allocate water. We assume that the sets of water users associated with different demand points are disjoints, that is, $K_{w} \cap K_{\tilde{w}}=\emptyset$ if $w \neq \tilde{w}$. Hence, $\left\{K_{w}\right\}_{w \in W}$ constitute a partition of $K$.

Let $I_{r}^{t}$ be the inflow in reservoir $r \in R$ during period $t \in T$. For each water user $k \in K_{w}, w \in W$, let $D_{w k}^{t}$ denote the total demand during period $t \in T$ and $b_{w k}^{t}$ be the economic return obtained by the demand point $w$ per unit of water allocated to the user $k$ during period $t$. Let $P_{w k}^{t}, w \in$ $W, k \in K_{w}, t \in T$, be an upper bound on the price, which the central authority can charge the demand point $w$ for the allocation of water to the water user $k$ during period $t$. We assume that $P_{w k}^{t} \leq b_{w k}^{t}, w \in W, k \in K_{w}, t \in T$, that is, the prices are never greater than the economic return of the corresponding water user.

Each reservoir $r \in R$ has a capacity and a minimum level of storage to be guaranteed at each period as required by the central authority. We denote $x_{r r}^{0}$ the amount of water available at the reservoir $r \in R$ at the beginning of the planning period. Moreover, the links between reservoirs and demand points represent either a river or a channel. Hence, in general, a link representing a river has a lower bound meaning a minimum downstream requirement and does not have an upper bound. This also applies to the water exiting the system. Conversely, a channel has an upper bound referring to its capacity and a lower bound equal to zero. We denote the lower bounds by $L$ and the upper bounds by $U$, with the proper indices in each case. For example, $L_{r r}$ and $U_{r r}$ denote, respectively, the minimum level of storage and the capacity of reservoir $r \in R$, while $L_{r w}$ and $U_{r w}$ are, respectively, zero and the capacity of the channel if there is a channel connecting the reservoir $r \in R$ and the demand point $w \in W$.

It is worth noting that, for a particular planning period, it cannot be guaranteed a priori that enough water will be available to satisfy all the needs. In fact, even if overall enough water was available during the planning horizon, depending on the characteristics of the water system, it is possible that not enough water would be available to ensure even the minimum level of storage and 
the minimum downstream requirements in some periods of the planning horizon. Also, water users can request more water than available in some periods. Therefore, to enforce minimum requirements and to maintain potential demands as mandatory demands that must be supplied would lead to an infeasible problem. However, in these cases where there is not enough water, it is even more important for the central authority to know how to distribute water according to its goals. Hence, the possibility of deficits is considered.

In order to formulate the multiobjective lexicographic multifollower bilevel optimization problem (MLMF-BP), we define the following upper level decision variables, which are controlled by the central authority:

$x_{r r}^{t}, r \in R, t \in T:$

storage at reservoir $r$ at the end of period $t$

$x_{r \tilde{r}}^{t}, r \in R, \tilde{r} \in R_{r}, t \in T:$

flow from reservoir $r$ to reservoir $\tilde{r}$ during period $t$

$x_{r w}^{t}, r \in R, w \in W_{r}, t \in T:$

$x_{r}^{t}, r \in R, t \in T:$

water sent from reservoir $r$ to demand point $w$ during period $t$

$s_{r r}^{t}, r \in R, t \in T:$

water leaving the system from reservoir $r$ during period $t$

storage deficit at reservoir $r$ at the end of period $t$

$s_{r \tilde{r}}^{t}, r \in R, \tilde{r} \in R_{r}, t \in T:$

minimum downstream requirement deficit from reservoir $r$ to reservoir $\tilde{r}$ during period $t$

$s_{r}^{t}, r \in R, t \in T:$

minimum downstream requirement deficit for the water exiting the system from reservoir $r$ during period $t$

$p_{w k}^{t}, w \in W, k \in K_{w}, t \in T: \quad$ price charged to the demand point $w$ per unit of water allocated to the water user $k$ during period $t$

Note that the variables $x_{r}^{t}$ and $s_{r}^{t}$ are only defined for those reservoirs $r$ from which the water can leave the system. Also, it must be noted that the aim of the central authority when using the variables $p_{w k}^{t}$ is not to maximize the return achieved from these prices but to influence the water allocation of demand points.

The lower level decision variables controlled by the demand point $w \in W$ are defined as follows:

$$
y_{w k}^{t}, k \in K_{w}, t \in T: \quad \text { water allocated to the water user } k \text { during period } t .
$$

As an illustration, a simple water system is shown at the top of Fig. 1. This consists of a sequential water system with two reservoirs, three demand points, and seven users. To help understand the evolution of the system over time and the variables involved, at the bottom of the same figure we have represented a diagram of the system over two periods. The variables associated to each arc are shown next to the arc.

\subsection{The multiobjective lexicographic multifollower bilevel optimization model}

The MLMF-BP can be formulated as follows:

$$
\min _{x, s, p, y} f_{1}^{1}=\sum_{t \in T} \sum_{r \in R}\left(s_{r r}^{t}+s_{r}^{t}+\sum_{\tilde{r} \in R_{r}} s_{r \tilde{r}}^{t}\right)
$$



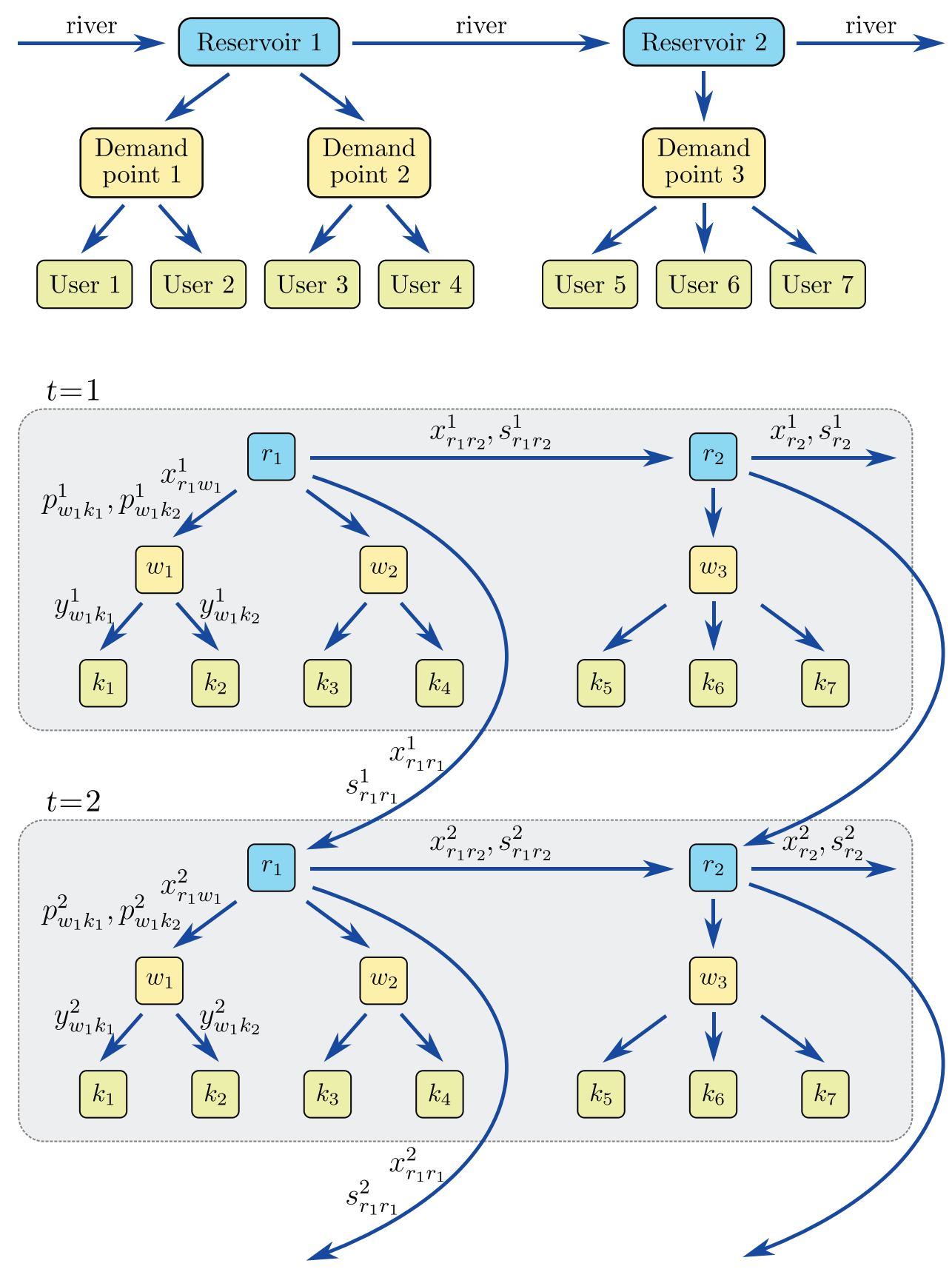

Fig. 1. A simple water system on top together with a representation of the flows and the variables involved when there are two periods of time, $T=\{1,2\}$. In this case, $R=\left\{r_{1}, r_{2}\right\}, W=\left\{w_{1}, w_{2}, w_{3}\right\}, K=\left\{k_{1}, \ldots, k_{7}\right\}, R_{r_{1}}=\left\{r_{2}\right\}$, $W_{r_{1}}=\left\{w_{1}, w_{2}\right\}$, and $K_{w_{1}}=\left\{k_{1}, k_{2}\right\}$. 


$$
\begin{aligned}
& \max _{x, s, p, y} \quad f_{1}^{2}=\sum_{t \in T} \sum_{w \in W} \sum_{k \in K_{w}} \frac{y_{w k}^{t}}{D_{w k}^{t}} \\
& \min _{x, s, p, y} \quad f_{1}^{3}=\sum_{t \in T} \sum_{w \in W} \sum_{k \in K_{w}} p_{w k}^{t}
\end{aligned}
$$

s.t.

$$
\begin{aligned}
& x_{r r}^{t-1}+I_{r}^{t}+\sum_{\tilde{r}: r \in R_{\tilde{r}}} x_{\tilde{r} r}^{t}=x_{r r}^{t}+x_{r}^{t}+\sum_{\tilde{r} \in R_{r}} x_{r \tilde{r}}^{t}+\sum_{w \in W_{r}} x_{r w}^{t}, \quad r \in R, t \in T \\
& x_{r r}^{t}+s_{r r}^{t} \geq L_{r r} \quad r \in R, t \in T \\
& x_{r}^{t}+s_{r}^{t} \geq L_{r} \quad r \in R, t \in T \\
& x_{r \tilde{r}}^{t}+s_{r \tilde{r}}^{t} \geq L_{r \tilde{r}} \quad r \in R, \tilde{r} \in R_{r}, t \in T \\
& x_{r r}^{t} \leq U_{r r} \quad r \in R, t \in T \\
& x_{r \tilde{r}}^{t} \leq U_{r \tilde{r}} \quad r \in R, \tilde{r} \in R_{r}, t \in T \\
& x_{r w}^{t} \leq U_{r w} \quad r \in R, w \in W_{r}, t \in T \\
& p_{w k}^{t} \leq P_{w k}^{t} \quad w \in W, k \in K_{w}, t \in T \\
& x_{r}^{t}, x_{r r}^{t}, x_{r \tilde{r}}^{t}, x_{r w}^{t}, s_{r}^{t}, s_{r r}^{t}, s_{r \tilde{r}}^{t} \geq 0 \quad r \in R, \tilde{r} \in R_{r}, w \in W_{r}, t \in T \\
& p_{w k}^{t} \geq 0 \quad w \in W, k \in K_{w}, t \in T,
\end{aligned}
$$

where, for each demand point $w \in W$, the variables $y_{w k}^{t}, k \in K_{w}, t \in T$, solve:

$$
\max _{y_{w}} f_{2 w}=\sum_{t \in T} \sum_{k \in K_{w}}\left(b_{w k}^{t}-p_{w k}^{t}\right) y_{w k}^{t}
$$

s.t.

$$
\begin{aligned}
& \sum_{k \in K_{w}} y_{w k}^{t} \leq \sum_{r \in R: w \in W_{r}} x_{r w}^{t}, \quad t \in T \\
& y_{w k}^{t} \leq D_{w k}^{t}, \quad k \in K_{w}, t \in T \\
& y_{w k}^{t} \geq 0, \quad k \in K_{w}, t \in T .
\end{aligned}
$$

The objective functions (3a)-(3c) lexicographically optimize the three ranked objectives of the central authority. Lexicographic optimality (Ehrgott, 2005) implies a ranking of the objectives. That is to say, it is assumed that the first objective is more important by far than the second objective, which in turn is more important by far than the third objective, and so on. Thus, the optimization 
of an objective is not considered until the optimality of the objectives, which precede it in importance, has been established. Concerning the MLMF-BP, the goal with the highest priority (3a) is to provide water to satisfy environmental and sustainability issues, that is, to meet the minimum requirements (minimum storage in reservoirs and minimum downstream requirements). Hence, the total deficit is minimized. If the central authority has to differentiate among those needs in case of not having enough water available to satisfy all of them, an additional weight coefficient can be associated with the deficit variables. This coefficient would allow us to emphasize the relevance of each need.

The second prioritized goal (3b) refers to the satisfaction of water users. In this paper, the concept of satisfaction proposed by Babel et al. (2005) as the ratio of water allocated over the demand is used. In addition, we propose to consider the utilitarian approach in which the goal is to maximize the total satisfaction of demand users measured by the sum of the individual satisfaction of each of them. As mentioned above, an additional weight coefficient can be associated with the individual satisfaction to emphasize its relevance. Other approaches that propose different strategies to deal with the allocation of resources across multiple users can be seen in Karsu and Erkan (2020) and references therein.

As mentioned above, the aim of the central authority in setting the prices is not to collect as much as possible but to be able to influence the allocation of water to the water users. Hence, from the, generally large, set of prices that provide the outcome sought by the central authority, we propose to select a set of low prices, and so a third prioritized goal (3c) is introduced which minimizes the sum of the prices. This will allow the demand points to be charged prices that together add up to as little as possible, always ensuring that the overall satisfaction represented by the second objective is maximized. In order to keep the model as simple as possible, within the complexity that is inherent in bilevel models, we have assumed that all the objective functions should be linear. Thus, the third objective function minimizes the total sum of prices instead of the total paid by users. The function $\sum_{t \in T} \sum_{w \in W} \sum_{k \in K_{w}} p_{w k}^{t} y_{w k}^{t}$ is not linear and we think that $f_{1}^{3}$ defined in (3c) fairly well captures the aim of the central authority. Nevertheless, it is important to note that other objective functions can be considered, which gives versatility to the proposed model. For instance, the central authority may want to minimize the highest price.

To continue with the description of the model, constraints (3d) ensure the conservation of flow. Constraints (3e) refer to the minimum level of storage in the reservoirs. Constraints (3f) refer to the minimum downstream requirements of the water leaving the water system. Constraints $(3 \mathrm{~g})$ refer to the minimum downstream requirements of the link connecting $r$ and $\tilde{r}$. Constraints (3h) ensure that the capacity of reservoirs is not exceeded. Constraints (3i) and (3j) allow us to guarantee that the capacity of each channel is not exceeded if the link corresponds to a channel, otherwise the upper bound is infinite. Constraints (3k) establish upper bounds on the prices $p_{w k}^{t}$, which seems appropriate to avoid unreasonable values for the prices. These upper bounds could be expressed, for instance, as a percentage of the economic return. Other price-related constraints could also be included in the model such as equal prices for certain water users even if they depend on different demand points. Constraints $(31)$ and $(3 \mathrm{~m})$ guarantee that all variables controlled by the central authority are nonnegative.

The lower level problem associated with the demand point $w \in W$ is defined by (3n)-(3q). After knowing the amount of water available, the manager of each demand point distributes the water to maximize the net economic return (revenues less costs) from water allocated to water users ( $3 \mathrm{n})$. 
Constraints (3o) ensure that at most the available water at demand point $w \in W$ is distributed among its water users $k \in K_{w}$. Constraints (3p) guarantee that no water user receives more than he/she demands. Constraints (3q) establish the nonnegativity of the lower level variables.

Problem (3) is an MLMF-BP with $|W|$ followers, where $|W|$ stands for the cardinality of $W$. Moreover, each follower problem involves only its own variables and the upper level variables. Hence, in accordance with the definition introduced in Calvete and Gale (2007), the followers are independent. By directly extending Theorem 3.1 from that paper to problem (3), this problem is equivalent to a multiobjective lexicographic bilevel optimization problem with one follower. The new lower level problem is obtained by considering the sum of the objective functions $f_{2 w}$ of the $|W|$ lower level problems as the objective function, and putting together all the lower level constraints. Consequently, problem (3) can be reformulated as follows:

$$
\operatorname{lex}\left[\min f_{1}^{1}, \max f_{1}^{2}, \min f_{1}^{3}\right]
$$

s.t.

$$
(3 \mathrm{~d})-(3 \mathrm{~m})
$$

where the variables $y_{w k}^{t}, w \in W, k \in K_{w}, t \in T$, solve

$$
\max _{y} f_{2}=\sum_{w \in W} \sum_{t \in T} \sum_{k \in K_{w}}\left(b_{w k}^{t}-p_{w k}^{t}\right) y_{w k}^{t}
$$

s.t.

$$
\begin{aligned}
& \sum_{k \in K_{w}} y_{w k}^{t} \leq \sum_{r \in R: w \in W_{r}} x_{r w}^{t}, \quad w \in W, t \in T \\
& y_{w k}^{t} \leq D_{w k}^{t}, \quad w \in W, k \in K_{w}, t \in T \\
& y_{w k}^{t} \geq 0, \quad w \in W, k \in K_{w}, t \in T,
\end{aligned}
$$

where lex means to lexicographically optimize the three objectives.

\subsection{Solving the $M L M F-B P$}

In this section, we propose to reformulate problem (4) as a single-level optimization problem using the KKT conditions of the lower level problem. For linear optimization, KKT conditions are necessary and sufficient for optimality. Hence, they can substitute the lower level problem (4c)-(4f) providing an equivalent problem.

Let us denote by $\pi_{w}^{t}, w \in W, t \in T$, and $\delta_{w k}^{t}, w \in W, k \in K_{w}, t \in T$, the dual variables associated with the constraints (4d) and (4e), respectively. Then, the dual problem of (4c)-(4f) is

$$
\min _{\pi, \delta} \sum_{t \in T} \sum_{w \in W}\left(\sum_{r \in R: w \in W_{r}} x_{r w}^{t}\right) \pi_{w}^{t}+\sum_{t \in T} \sum_{w \in W} \sum_{k \in K_{w}} D_{w k}^{t} \delta_{w k}^{t}
$$


s.t.

$$
\begin{aligned}
& \pi_{w}^{t}+\delta_{w k}^{t} \geq b_{w k}^{t}-p_{w k}^{t}, \quad w \in W, k \in K_{w}, t \in T \\
& \pi_{w}^{t} \geq 0, \quad w \in W, t \in T \\
& \delta_{w k}^{t} \geq 0, \quad w \in W, k \in K_{w}, t \in T .
\end{aligned}
$$

Therefore, in addition to the feasibility of primal and dual solutions (constraints (4d)-(4f), (5b)(5d), the KKT conditions are

$$
\begin{aligned}
& \left(\pi_{w}^{t}+\delta_{w k}^{t}-b_{w k}^{t}+p_{w k}^{t}\right) y_{w k}^{t}=0, \quad w \in W, k \in K_{w}, t \in T \\
& \left(\sum_{r \in R: w \in W_{r}} x_{r w}^{t}-\sum_{k \in K_{w}} y_{w k}^{t}\right) \pi_{w}^{t}=0, \quad w \in W, t \in T \\
& \left(D_{w k}^{t}-y_{w k}^{t}\right) \delta_{w k}^{t}=0, \quad w \in W, k \in K_{w}, t \in T .
\end{aligned}
$$

Nonlinear constraints (6a)-(6c) can be linearized by introducing binary variables $v_{w k}^{t}, v_{w}^{t}$, and $\tilde{v}_{w k}^{t}$ as

$$
\begin{aligned}
& y_{w k}^{t} \leq M_{w k}^{t} v_{w k}^{t}, \quad w \in W, k \in K_{w}, t \in T \\
& \pi_{w}^{t}+\delta_{w k}^{t}-b_{w k}^{t}+p_{w k}^{t} \leq N_{w k}^{t}\left(1-v_{w k}^{t}\right), \quad w \in W, k \in K_{w}, t \in T \\
& \pi_{w}^{t} \leq M_{w}^{t} v_{w}^{t}, \quad w \in W, t \in T \\
& \sum_{r \in R: w \in W_{r}} x_{r w}^{t}-\sum_{k \in K_{w}} y_{w k}^{t} \leq N_{w}^{t}\left(1-v_{w}^{t}\right), \quad w \in W, t \in T \\
& \delta_{w k}^{t} \leq \tilde{M}_{w k}^{t} \tilde{v}_{w k}^{t}, \quad w \in W, k \in K_{w}, t \in T \\
& D_{w k}^{t}-y_{w k}^{t} \leq \tilde{N}_{w k}^{t}\left(1-\tilde{v}_{w k}^{t}\right), \quad w \in W, k \in K_{w}, t \in T \\
& v_{w k}^{t}, \tilde{v}_{w k}^{t} \in\{0,1\}, \quad w \in W, k \in K_{w}, t \in T \\
& v_{w}^{t} \in\{0,1\}, \quad w \in W, t \in T,
\end{aligned}
$$

where $M_{w k}^{t}, N_{w k}^{t}, M_{w}^{t}, N_{w}^{t}, \tilde{M}_{w k}^{t}, \tilde{N}_{w k}^{t}$ are big enough constants.

Substituting the lower level problem in (4) by its KKT conditions, we obtain the equivalent singlelevel multiobjective lexicographic mixed-integer problem:

$\operatorname{lex}\left[\min f_{1}^{1}, \max f_{1}^{2}, \min f_{1}^{3}\right]$

s.t.

$$
\text { (3d)-(3m), (4d)-(4f), (5b)-(5d), (7a)-(7h). }
$$


Issues related to the choice of appropriate values of these big enough constants have been dealt with in Kleinert et al. (2020) and Pineda and Morales (2019). As cited in Kleinert et al. (2020), our results strongly indicate that problem-specific bounds on the lower level's dual variables need to be investigated if the given bilevel problem is going to be solved using the KKT approach combined with the classical big- $M$ linearization of KKT complementarity conditions. Taking into account the characteristics of the primal and dual problems, the following theorem allows us to derive tight upper bounds.

Theorem 1. For problem (8), valid constants are

$$
\begin{array}{ll}
\text { constraints }(7 a): & M_{w k}^{t}=D_{w k}^{t} \\
\text { constraints }(7 b): & N_{w k}^{t}=b_{w k}^{t}+\max _{\tilde{k} \in K_{w}}\left\{b_{w \tilde{k}}^{t}\right\} \\
\text { constraints }(7 c): & M_{w}^{t}=\max _{\tilde{k} \in K_{w}}\left\{b_{w \tilde{k}}^{t}\right\} \\
\text { constraints }(7 d): & N_{w}^{t}=\sum_{k \in K_{w}} D_{w k}^{t} \\
\text { constraints }(7 e): & \tilde{M}_{w k}^{t}=b_{w k}^{t} \\
\text { constraints }(7 f): & \tilde{N}_{w k}^{t}=D_{w k}^{t}
\end{array}
$$

Proof. According to constraints (4e), $y_{w k}^{t} \leq D_{w k}^{t}$. Hence $M_{w k}^{t}=D_{w k}^{t}, \tilde{N}_{w}^{t}=D_{w k}^{t}$ are valid constants for constraints (7a) and (7f), respectively.

Problem (5) is a minimization problem with nonnegative variables and nonnegative objective function coefficients. Moreover, the right-hand side of each constraint (5b) is at most $b_{w k}^{t}$ since $p_{w k}^{t} \geq 0$. Hence, in the optimal solution of problem (5), $\delta_{w k}^{t} \leq b_{w k}^{t}$, for all $w \in W, k \in K_{w}, t \in T$, whatever the value of the variables $\pi_{w}^{t}$ which guarantee a feasible solution. Note that from each feasible solution having $\delta_{w k}^{t}>b_{w k}^{t}$ for some index values, a feasible solution with a lower value of the objective function (5a) can be obtained by making $\delta_{w k}^{t}=b_{w k}^{t}$. Hence, $\tilde{M}_{w k}^{t}=b_{w k}^{t}$ is a valid constant for constraints (7e).

On the other hand, for each $w \in W, t \in T$, the variable $\pi_{w}^{t} \leq \max _{\tilde{k} \in K_{w}}\left\{b_{w \tilde{k}}^{t}\right\}$ in the optimal solution of problem (5). Otherwise, using the same argument as above, $\pi_{w}^{t}=\max _{\tilde{k} \in K_{w}}\left\{b_{w \tilde{k}}^{t}\right\}$ and the corresponding values of $\delta_{w k}^{t}$ would provide a feasible solution with a lower value of the objective function (5a). Hence, $M_{w}^{t}=\max _{\tilde{k} \in K_{w}}\left\{b_{w \tilde{k}}^{t}\right\}$ is a valid constant for constraints (7c).

As a consequence of previous bounds on $\delta_{w k}^{t}$ and $\pi_{w}^{t}$ and taking into account that $p_{w k}^{t} \leq P_{w k}^{t} \leq$ $b_{w k}^{t}$, we can conclude that $N_{w k}^{t}=b_{w k}^{t}+\max _{\tilde{k} \in K_{w}}\left\{b_{w \tilde{k}}^{t}\right\}$ is a valid constant for constraints (7b).

Finally, considering the optimal solution of problem (3), it is always possible to find an optimal solution in which no demand point receives more water than it can allocate to its water users. Thus, for each $w \in W, t \in T$, we have $\sum_{r \in R: w \in W_{r}} x_{r w}^{t} \leq \sum_{k \in K_{w}} D_{k}^{t}$. Hence, $N_{w}^{t}=\sum_{k \in K_{w}} D_{w k}^{t}$ is a valid constant for constraints $(7 \mathrm{~d})$.

\section{Computational experiments}

The aim of this section is to present and discuss the computational experiments carried out to illustrate the differences between the optimal water-allocation strategies depending on the water 
available and the prices charged, and to show the efficiency of the procedure to solve the MLMFBP. The numerical experiments have been performed on a PC Intel Core i7-6700 with 3.4 GHz, 32.0 GB of RAM, and Windows 10 64-bit as the operating system. We have solved problem (8) using IBM ILOG CPLEX 12.9.0 with the default settings and the specific tools for multiobjective optimization. The CPLEX stopping criterion has been always set at 7200 seconds.

In addition to solving the MLMF-BP, in these experiments we have also solved what is called the relaxed problem in bilevel optimization. This is a single-level model in which the central authority controls every allocation of water, that is, demand point managers are not taken into account in the decision process. It can be formulated as

$$
\begin{aligned}
& \operatorname{lex}\left[\min f_{1}^{1}, \max f_{1}^{2}\right] \\
& \text { s.t. } \\
& \quad(3 \mathrm{~d})-(3 \mathrm{j}),(3 \mathrm{l}),(4 \mathrm{~d})-(4 \mathrm{f}) .
\end{aligned}
$$

The third objective function $f_{1}^{3}$ and the constraints $(3 \mathrm{k})$ and $(3 \mathrm{~m})$ have been omitted because they force all the prices $p_{w k}^{t}$ to be zero. Needless to say, when demand points are not accounted for, it does not make sense to charge prices.

On the other hand, the optimal value of $f_{1}^{1}$ in the bilevel problem (3) coincides with the optimal value of the same function in the relaxed problem (9) (both aim to minimize the total deficit, which is controlled by the upper level decision maker, and do not take into account how the demand points are served). Hence, when comparing problems (3) and (9) the main interest lies in the comparison of the upper level objective function $f_{1}^{2}$. Problem (9) provides a lower bound on $f_{1}^{2}$ of the MLMF-BP, but it does not reflect what actually happens if there exists a hierarchical structure with two levels of decision. Note that once the demand point managers know the value of the upper level variables provided by the optimal solution of the relaxed problem, they assume their role and distribute the allocated water according to their goal. The bilevel feasible solution thus obtained (from now on called the suboptimal solution) is not necessarily optimal for the MLMF-BP. The use of prices can help the central authority to achieve its goals, obtaining objective function values closer to the optimal values of problem (9).

\subsection{Set of instances}

Most papers in the literature consider cases of study corresponding to small water systems with only one or two reservoirs and they do not provide raw data. When some data are available, their sizes depend largely on the system under consideration. Hence, to have a wide range of scenarios, which allow us to check the versatility of the model as well as to assess the computational time required to solve the model, we have generated six water systems that represent either water systems usually presented in the literature or more complex systems that combine them. They are shown in Fig. 2. Instances WS1 and WS2 consist of a single river, instances WS3 and WS4 consist of a main river with two tributaries, and instances WS5 and WS6 consist of two main rivers connected, respectively, by one and two channels. The number of reservoirs ranges from 4 to 7 , the number of demand points ranges from 7 to 12, and the number of water users ranges from 74 to 142 . For each instance, we have considered three planning horizons, with 12, 52, and 365 periods. 


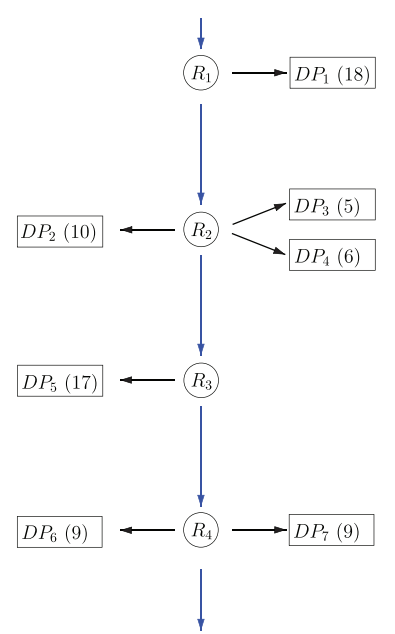

(a) WS1

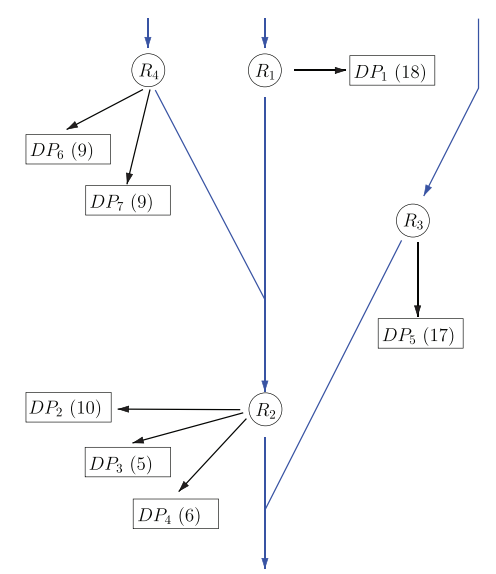

(c) WS3

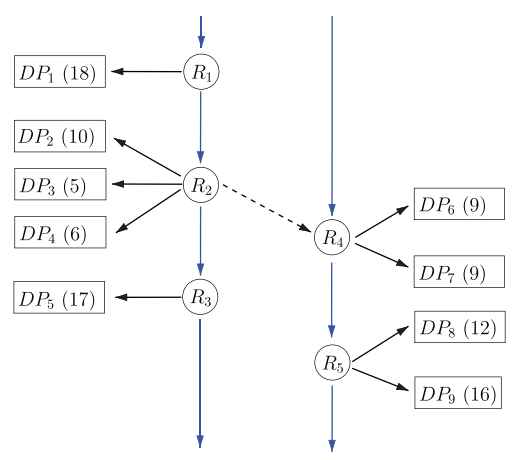

(e) WS5

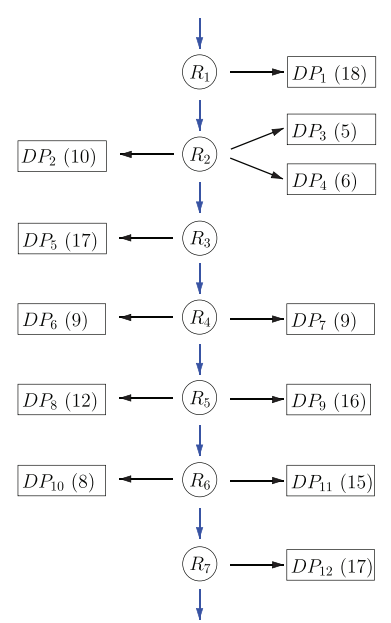

(b) WS2

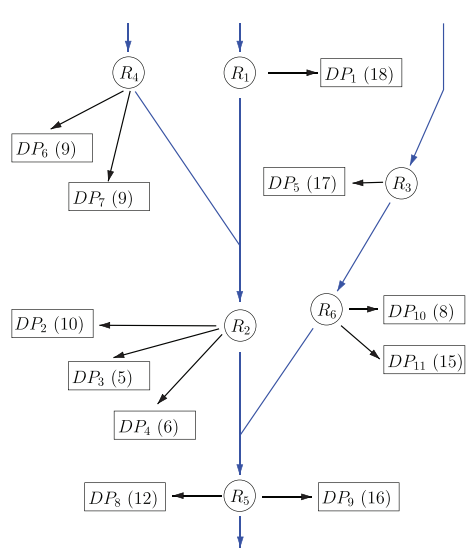

(d) WS4

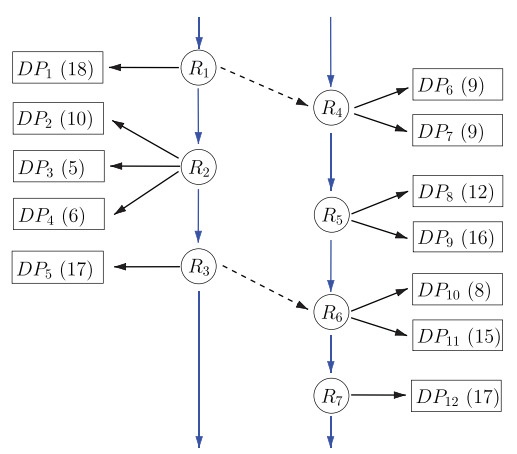

(f) WS6

Fig. 2. Schemes of the water systems of the instances. The numbers in brackets refer to number of water users associated to the corresponding demand point. 
Table 1

Randomly generated inflows for $T=12$

\begin{tabular}{rllllllllllll}
\hline & 1 & 2 & 3 & 4 & 5 & 6 & 7 & 8 & 9 & 10 & 11 & 12 \\
\hline$\left(I_{r}^{t}\right)_{1}$ & 29,220 & 25,775 & 19,998 & 13,243 & 7337 & 3403 & 3680 & 6633 & 12,401 & 19,018 & 25,269 & 29,050 \\
$\left(I_{r}^{t}\right)_{2}$ & 21,841 & 19,501 & 15,002 & 10,015 & 5312 & 3069 & 2577 & 4801 & 9259 & 14,237 & 18,914 & 21,947 \\
$\left(I_{r}^{t}\right)_{3}$ & 14,696 & 12,816 & 10,083 & 6669 & 3666 & 1911 & 1856 & 3228 & 6167 & 9730 & 12,491 & 14,724 \\
\hline
\end{tabular}

Three yearly inflows (365 periods) have been generated from the following expressions:

$$
\begin{aligned}
& \left(I_{r}^{t}\right)_{1}=450 \cos \frac{2 \pi t}{365}+\operatorname{random}(500,600), \quad t=1, \ldots, 365 \\
& \left(I_{r}^{t}\right)_{2}=0.75 \times\left(450 \cos \frac{2 \pi t}{365}+\operatorname{random}(500,600)\right), \quad t=1, \ldots, 365 \\
& \left(I_{r}^{t}\right)_{3}=0.5 \times\left(450 \cos \frac{2 \pi t}{365}+\operatorname{random}(500,600)\right), \quad t=1, \ldots, 365
\end{aligned}
$$

Most streams display annual variation due to seasonal changes. In the Mediterranean countries the lowest flows often occur near the end of the spring and during the summer. These sinusoidal functions can represent this behavior.

From the values generated by expression (10), when 12 (respectively, 52) periods are considered, the first 360 (364) values are added up in groups of 30 (7) data each. Table 1 shows the inflow values when 12 periods are considered. Note that less water is available in the central periods. Moreover, distinct scenarios of flow are obtained by multiplying randomly generated inflows by a coefficient $\beta$ which ranges from 0.1 to 1 in order to simulate variations from a major drought scenario to a high water scenario. For $\beta=0.8,0.9$, and 1 the same results are obtained as for $\beta=0.7$, since there is enough water available to satisfy total demand. Thus, these values of $\beta$ will be omitted from now on. The instance WS1 uses $\left(I_{r}^{t}\right)_{2}$; the instance WS2 uses $\left(I_{r}^{t}\right)_{1}$; the instances WS3 and WS4 use $\left(I_{r}^{t}\right)_{1}$ in $R_{1},\left(I_{r}^{t}\right)_{2}$ in $R_{3}$, and $\left(I_{r}^{t}\right)_{3}$ in $R_{4}$; and the instances WS5 and WS6 use $\left(I_{r}^{t}\right)_{2}$ in $R_{1}$ and $\left(I_{r}^{t}\right)_{3}$ in $R_{4}$.

For each reservoir $r \in R$, its capacity $U_{r r}$ has been randomly generated as an integer in [5000, 50, 000], the minimum level of storage $L_{r r}=0.01 U_{r r}$, and the amount of water available at the beginning of the planning period $x_{r r}^{0}=\beta\left(U_{r r}+L_{r r}\right) / 2$. Regarding the water users, $50 \%$ have a daily demand randomly generated in [1,2]; 30\% have a daily demand randomly generated in [2, 6]; and $20 \%$ have a daily demand randomly generated in $[6,10]$. The daily minimum downstream requirement has been set at 10, and the maximum daily capacity of the channels has been set at 2000 . Daily data are added up, as done for the inflows, depending on the periods of the planning horizon. The upper bounds $U_{r w}$ are infinite. Table 2 displays the capacity of each reservoir, the number of demand points and of water users associated to it, and the total demand of the water users in each period of time when 12 periods are considered. Table 3 displays the total water available in each water system depending on the value of $\beta$, as well as the total demand when $T=12$.

For each water user $k \in K_{w}, w \in W$, the economic return $b_{w k}^{t}$ is randomly generated as an integer value in the interval $[10,30]$ and it is same in all the periods. To assess the effect of varying the bounds $P_{w k}^{t}$, we have selected those bounds as a percentage of the economic return, that is, $P_{w k}^{t}=$ $\alpha b_{w k}^{t}$, where $\alpha=0,0.25,0.5,0.75$. Note that when $\alpha=0$ no prices are charged.

For these instances, the MLMF-BP has been solved for each combination of water system, values of $\alpha$ and $\beta$, and length of the planning horizon, that is, 720 problems have been solved. Moreover, 
Table 2

For each reservoir: Its capacity, the demand points associated with it, the number of water users corresponding to each demand point, and the total demand of these water users in each period of time when $T=12$

\begin{tabular}{|c|c|c|c|c|c|c|c|c|c|c|c|c|c|c|c|}
\hline & \multirow[b]{2}{*}{$U_{r r}$} & \multirow[b]{2}{*}{$\mathrm{DP}$} & \multirow[b]{2}{*}{$\left|K_{w}\right|$} & \multicolumn{12}{|c|}{ Total demand of each demand point in each period of time } \\
\hline & & & & 1 & 2 & 3 & 4 & 5 & 6 & 7 & 8 & 9 & 10 & 11 & 12 \\
\hline$R_{1}$ & 35,469 & 1 & 18 & 1436 & 1523 & 1556 & 1707 & 1786 & 1856 & 1875 & 1802 & 1700 & 1584 & 1501 & 1435 \\
\hline \multirow[t]{3}{*}{$R_{2}$} & 38,598 & 2 & 10 & 979 & 1012 & 1031 & 1094 & 1121 & 1163 & 1168 & 1158 & 1090 & 1034 & 999 & 992 \\
\hline & & 3 & 5 & 714 & 721 & 731 & 795 & 804 & 826 & 843 & 794 & 802 & 743 & 723 & 712 \\
\hline & & 4 & 6 & 359 & 367 & 398 & 441 & 476 & 491 & 497 & 476 & 444 & 404 & 377 & 350 \\
\hline$R_{3}$ & 36,099 & 5 & 17 & 1629 & 1707 & 1768 & 1925 & 1985 & 2040 & 2016 & 1981 & 1892 & 1805 & 1693 & 1655 \\
\hline \multirow[t]{2}{*}{$R_{4}$} & 13,545 & 6 & 9 & 860 & 880 & 904 & 961 & 1017 & 1031 & 1018 & 1024 & 959 & 939 & 905 & 850 \\
\hline & & 7 & 9 & 866 & 880 & 937 & 969 & 1020 & 1031 & 1032 & 1015 & 977 & 931 & 868 & 848 \\
\hline \multirow[t]{2}{*}{$R_{5}$} & 42,588 & 8 & 12 & 1000 & 1049 & 1099 & 1213 & 1250 & 1282 & 1295 & 1264 & 1198 & 1110 & 1070 & 1036 \\
\hline & & 9 & 16 & 1729 & 1778 & 1865 & 2001 & 2103 & 2186 & 2162 & 2101 & 2005 & 1910 & 1782 & 1711 \\
\hline \multirow[t]{2}{*}{$R_{6}$} & 21,063 & 10 & 8 & 852 & 894 & 951 & 999 & 1046 & 1081 & 1098 & 1087 & 996 & 939 & 910 & 840 \\
\hline & & 11 & 15 & 1281 & 1307 & 1407 & 1472 & & 1611 & & 1553 & 1470 & 1434 & 1338 & 1284 \\
\hline$R_{7}$ & 11,760 & 12 & 17 & 1658 & 1744 & 1844 & 1993 & 2088 & 2156 & 2183 & 2110 & 2002 & 1870 & 1717 & 1670 \\
\hline
\end{tabular}

Table 3

For each water system: The inflows involved, total water available depending on the value of $\beta$ (inflow plus water available at the reservoirs at the beginning of the planning period) and total demand of its water users when $T=12$

\begin{tabular}{|c|c|c|c|c|c|c|c|c|c|}
\hline & & \multicolumn{7}{|l|}{$\beta$} & \multirow[b]{2}{*}{ Demand } \\
\hline & & 0.1 & 0.2 & 0.3 & 0.4 & 0.5 & 0.6 & 0.7 & \\
\hline WS1 & $\left(I_{r}^{t}\right)_{2}$ & 20,895 & 41,790 & 62,684 & 83,579 & 104,474 & 125,369 & 146,263 & 91,708 \\
\hline WS2 & $\left(I_{r}^{t}\right)_{1}$ & 29,558 & 59,117 & 88,675 & 118,234 & 147,792 & 177,350 & 206,909 & 180,945 \\
\hline WS3 & $\left(I_{r}^{t}\right)_{1},\left(I_{r}^{t}\right)_{2},\left(I_{r}^{t}\right)_{3}$ & 50,201 & 100,402 & 150,603 & 200,805 & 251,006 & 301,207 & 351,408 & 91,708 \\
\hline WS4 & $\left(I_{t}^{t}\right)_{1},\left(I_{r}^{t}\right)_{2},\left(I_{t}^{t}\right)_{3}$ & 53,416 & 106,831 & 160,247 & 213,662 & 267,078 & 320,493 & 373,909 & 157,911 \\
\hline WS5 & $\left(I_{r}^{t}\right)_{2},\left(I_{r}^{t}\right)_{3}$ & 32,849 & 65,698 & 98,548 & 131,397 & 164,246 & 197,095 & 229,944 & 128,907 \\
\hline WS6 & $\left(I_{r}^{t}\right)_{2},\left(I_{r}^{t}\right)_{3}$ & 34,507 & 69,014 & 103,520 & 138,027 & 172,534 & 207,041 & 241,548 & 180,945 \\
\hline
\end{tabular}

the relaxed problem has been solved for each water system and value of $\beta$ from 0.1 to 0.7 , that is, 126 problems, and for the values of the upper level variables another 126 lower level problems have been solved to obtain the suboptimal solution.

The large number of problems solved makes it unwieldy to analyze every optimal solution obtained. To have an insight into the optimal solutions of the relaxed problem, the bilevel feasible solution associated with it (suboptimal solution) and the evolution of the optimal solution of the bilevel problem when $\alpha$ increases, we have selected the instance WS1 with $\beta=0.1$ (major drought scenario) and $T=12$. Since $f_{1}^{1}=0$, we pay attention to how water users are served in accordance with those solutions. We define

- $K_{0}$ as the number of water users who do not receive water in any period;

- $K_{1}$ as the number of water users who receive all their demand in all periods;

- $K_{\max }$ as the maximum, over the periods, of the water users who receive water. 
Table 4

Instance WS1 with $\beta=0.1$ and $T=12$ : Water users' satisfaction with respect to the models considered

\begin{tabular}{|c|c|c|c|c|c|c|c|c|c|c|c|c|c|c|c|c|}
\hline \multirow[b]{2}{*}{ DP } & \multirow[b]{2}{*}{$\left|K_{w}\right|$} & \multicolumn{3}{|c|}{ Relaxed } & \multicolumn{3}{|c|}{ Suboptimal } & \multicolumn{3}{|c|}{ Bilevel, $\alpha=0$} & \multicolumn{3}{|c|}{ Bilevel, $\alpha=0.25$} & \multicolumn{3}{|c|}{ Bilevel, $\alpha=0.5$} \\
\hline & & $\overline{K_{0}}$ & $K_{1}$ & $K_{\max }$ & $\overline{K_{0}}$ & $K_{1}$ & $K_{\max }$ & $\overline{K_{0}}$ & $K_{1}$ & $\overline{K_{\max }}$ & $\overline{K_{0}}$ & $K_{1}$ & $K_{\max }$ & $\overline{K_{0}}$ & $K_{1}$ & $K_{\max }$ \\
\hline 1 & 18 & 7 & 4 & 11 & 10 & 2 & 8 & 11 & 7 & 7 & 7 & 6 & 11 & 6 & 6 & 12 \\
\hline 2 & 10 & 5 & 0 & 5 & 8 & 0 & 2 & 1 & 0 & 9 & 2 & 2 & 8 & 5 & 3 & 5 \\
\hline 3 & 5 & 3 & 0 & 2 & 4 & 0 & 1 & 5 & 0 & 0 & 5 & 0 & 0 & 3 & 0 & 2 \\
\hline 4 & 6 & 1 & 1 & 5 & 2 & 1 & 4 & 0 & 2 & 6 & 0 & 5 & 6 & 1 & 3 & 5 \\
\hline 5 & 17 & 7 & 3 & 10 & 12 & 1 & 5 & 6 & 0 & 11 & 11 & 4 & 6 & 8 & 5 & 9 \\
\hline 6 & 9 & 4 & 1 & 5 & 8 & 0 & 1 & 9 & 0 & 0 & 7 & 2 & 2 & 4 & 2 & 5 \\
\hline 7 & 9 & 3 & 0 & 6 & 7 & 1 & 2 & 8 & 1 & 1 & 7 & 2 & 2 & 4 & 3 & 5 \\
\hline
\end{tabular}

The first and second columns in Table 4 display the demand point and the number of its water users. The following columns show the values of $K_{0}, K_{1}$, and $K_{\max }$ for each of the above-mentioned solutions. The values corresponding to the bilevel problem with $\alpha=0.75$ have been omitted because its optimal solution coincides with the optimal solution of the relaxed problem. Looking at these values, we can observe that the solutions are very distinct, which shows that problems (9) and (3) model very different systems, a centralized system (the relaxed problem) and a decentralized system that takes into account the reaction of the users (the bilevel problem), respectively. On the other hand, as the value of $\alpha$ increases the values $K_{0}, K_{1}$, and $K_{\max }$ of the bilevel solution become more and more similar to the values of the relaxed problem solution. The suboptimal solution provides the largest values of $K_{0}$, and the lowest of $K_{\max }$.

In the following subsections, we summarize the results of the complete experiment to give an overview of the degree to which the central authority goals are met depending on the water availability scenario. This can help decision makers to better understand the interactions among environmental requirements, demand points, water users, and prices.

\subsection{Assessing the model in terms of number of variables and computing time}

The aim of this section is to provide general information about the complexity of the model measured by the number of variables and constraints involved in the models and by the average computing time required for solving the problems. Table 5 displays these results. The first and second columns show the number of periods of the planning period and the water system. The third column displays the number of variables of the relaxed problem (9). The fourth and fifth columns show, respectively, the total number of variables in the bilevel model (8) and how many of them are binary variables. The sixth and seventh columns display the number of constraints of the relaxed and bilevel problems, respectively.

Finally, the two last columns display the average of the CPU time in seconds of the 7 (28) runs corresponding to the relaxed (bilevel) model. Regarding the average times, as could be expected, the relaxed model, which does not involve integer variables, is solved in very short computing times. Concerning the bilevel model, it is worth pointing out that the computing times are quite small when $T=12$ or $T=52$ (less than 3 and 30 seconds, respectively). Moreover, although these times increase, especially for the more complex water systems 2 and 6 , when $T=365$, reaching an aver- 
H.I. Calvete et al. / Intl. Trans. in Op. Res. 0 (2021) 1-30

Table 5

For each instance and planning period, the number of variables and constraints, and the average CPU time

\begin{tabular}{|c|c|c|c|c|c|c|c|c|}
\hline & & \multicolumn{3}{|c|}{ \# of variables } & \multicolumn{2}{|c|}{ \# of constraints } & \multicolumn{2}{|c|}{ Average CPU } \\
\hline & & Relaxed & Bilevel & $(0-1)$ & Relaxed & Bilevel & Relaxed & Bilevel \\
\hline \multirow[t]{6}{*}{$T=12$} & WS1 & 1236 & 4956 & 1860 & 2208 & 6816 & 0.06 & 0.53 \\
\hline & WS2 & 2328 & 9432 & 3552 & 4176 & 12,984 & 0.09 & 1.90 \\
\hline & WS3 & 1212 & 4932 & 1860 & 2184 & 6792 & 0.05 & 0.50 \\
\hline & WS4 & 2040 & 8304 & 3132 & 3672 & 11,436 & 0.09 & 1.03 \\
\hline & WS5 & 1668 & 6780 & 2556 & 3000 & 9336 & 0.08 & 0.82 \\
\hline & WS6 & 2352 & 9456 & 3552 & 4200 & 13,008 & 0.09 & 1.61 \\
\hline \multirow[t]{6}{*}{$T=52$} & WS1 & 9568 & 29,536 & 5356 & 21,476 & 8060 & 0.12 & 5.37 \\
\hline & WS2 & 18,096 & 56,264 & 10,088 & 40,872 & 15,392 & 0.23 & 20.06 \\
\hline & WS3 & 9464 & 29,432 & 5252 & 21,372 & 8060 & 0.13 & 3.77 \\
\hline & WS4 & 15,912 & 49,556 & 8840 & 35,984 & 13,572 & 0.19 & 16.86 \\
\hline & WS5 & 13,000 & 40,456 & 7228 & 29,380 & 11,076 & 0.17 & 11.72 \\
\hline & WS6 & 18,200 & 56,368 & 10,192 & 40,976 & 15,392 & 0.22 & 27.64 \\
\hline \multirow[t]{6}{*}{$T=365$} & WS1 & 37,595 & 150,745 & 56,575 & 67,160 & 207,320 & 1.49 & 246.23 \\
\hline & WS2 & 70,810 & 286,890 & 108,040 & 127,020 & 394,930 & 3.47 & 1734.69 \\
\hline & WS3 & 36,865 & 150,015 & 56,575 & 66,430 & 206,590 & 2.38 & 268.60 \\
\hline & WS4 & 62,050 & 252,580 & 95,265 & 111,690 & 347,845 & 2.75 & 778.01 \\
\hline & WS5 & 50,735 & 206,225 & 77,745 & 91,250 & 283,970 & 1.76 & 895.76 \\
\hline & WS6 & 71,540 & 287,620 & 108,040 & 127,750 & 395,660 & 2.93 & 1346.72 \\
\hline
\end{tabular}

age computing time of almost 30 minutes, these times can be considered competitive taking into account both the number of variables and constraints involved. The detailed results regarding the computing time are presented in the Appendix. It is worth mentioning at this point that, even if the bilevel problem is much more complicated to solve, it is the appropriate model when modeling a decentralized system that takes into account the reaction of the users, as noted when describing the MLMF-BP. In the previous and following sections, we show that the suboptimal solution is not necessarily optimal for the MLMF-BP. In fact, it can be very far from the bilevel optimal solution, and a priori it is not possible to know what extent.

\subsection{Degree to which the goals of the central authority are met}

For all the instances and available water, it is possible to satisfy environmental and sustainability issues, that is, the minimum needs are always met and thus $f_{1}^{1}$ is always equal to zero. For small values of $\beta$ it is not possible to satisfy the demand of all the water users, even if the reaction of the demand points is not taken into account. The level of satisfaction of demand users, and so the second goal of the central authority, depends largely on the water system analyzed. To satisfy all the water users in all periods requires $\beta \geq 0.3$ for instance WS 3 and $\beta \geq 0.4$ for instance WS4, which have a similar structure with a river and two tributaries. Instances WS1 and WS5 require $\beta \geq 0.5$. Finally, the most demanding systems from the point of view of the computing time required are also the instances that need a larger value of $\beta$ to provide total satisfaction. These are WS6 that requires $\beta \geq 0.6$ and WS2 that requires $\beta \geq 0.7$. In these cases, the demand of all the 
Table 6

The satisfaction index $I$ for $T=12$

\begin{tabular}{|c|c|c|c|c|c|c|c|}
\hline & \multirow[b]{2}{*}{$\beta$} & \multicolumn{2}{|l|}{ Relaxed } & \multicolumn{4}{|l|}{ Bilevel } \\
\hline & & Optimal & Suboptimal & $\alpha=0$ & $\alpha=0.25$ & $\alpha=0.5$ & $\alpha=0.75$ \\
\hline \multirow[t]{4}{*}{ WS1 } & 0.1 & 0.42 & 0.19 & 0.27 & 0.35 & 0.41 & $=$ \\
\hline & 0.2 & 0.69 & 0.43 & 0.53 & 0.60 & 0.66 & $=$ \\
\hline & 0.3 & 0.85 & 0.69 & 0.75 & 0.79 & 0.83 & $=$ \\
\hline & 0.4 & 0.94 & 0.87 & 0.92 & 0.93 & $=$ & $=$ \\
\hline \multirow[t]{6}{*}{ WS2 } & 0.1 & 0.33 & 0.16 & 0.22 & 0.30 & $=$ & $=$ \\
\hline & 0.2 & 0.59 & 0.31 & 0.40 & 0.49 & 0.56 & $=$ \\
\hline & 0.3 & 0.73 & 0.49 & 0.57 & 0.64 & 0.70 & $=$ \\
\hline & 0.4 & 0.84 & 0.68 & 0.73 & 0.78 & 0.82 & $=$ \\
\hline & 0.5 & 0.91 & 0.81 & 0.87 & 0.89 & $=$ & $=$ \\
\hline & 0.6 & 0.97 & 0.94 & 0.96 & $=$ & $=$ & $=$ \\
\hline \multirow[t]{2}{*}{ WS3 } & 0.1 & 0.72 & 0.49 & 0.57 & 0.63 & 0.69 & $=$ \\
\hline & 0.2 & 0.95 & 0.88 & 0.92 & 0.93 & $=$ & $=$ \\
\hline \multirow[t]{3}{*}{ WS4 } & 0.1 & 0.59 & 0.33 & 0.41 & 0.49 & 0.56 & $=$ \\
\hline & 0.2 & 0.84 & 0.68 & 0.74 & 0.78 & 0.82 & $=$ \\
\hline & 0.3 & 0.96 & 0.92 & 0.95 & $=$ & $=$ & $=$ \\
\hline \multirow[t]{4}{*}{ WS5 } & 0.1 & 0.44 & 0.19 & 0.27 & 0.35 & 0.43 & $=$ \\
\hline & 0.2 & 0.72 & 0.46 & 0.55 & 0.62 & 0.68 & $=$ \\
\hline & 0.3 & 0.87 & 0.72 & 0.78 & 0.82 & 0.86 & $=$ \\
\hline & 0.4 & 0.96 & 0.91 & 0.95 & $=$ & $=$ & $=$ \\
\hline \multirow[t]{5}{*}{ WS6 } & 0.1 & 0.35 & 0.16 & 0.23 & 0.31 & $=$ & \\
\hline & 0.2 & 0.62 & 0.35 & 0.44 & 0.52 & 0.59 & $=$ \\
\hline & 0.3 & 0.78 & 0.56 & 0.63 & 0.69 & 0.75 & $=$ \\
\hline & 0.4 & 0.88 & 0.75 & 0.80 & 0.84 & 0.87 & $=$ \\
\hline & 0.5 & 0.95 & 0.89 & 0.93 & $=$ & $=$ & $=$ \\
\hline
\end{tabular}

water users is satisfied without the need of charging a price to the demand points. Needless to say, when there is "enough" water there are no conflicts. It is not important whether it is the central authority or the demand points that take control of the allocation of water to water users because all the objective functions achieve their best theoretical values $\left(f_{1}^{1}=0, f_{1}^{2}=|T| \times|K|, f_{1}^{3}=0\right.$, $\left.f_{2}=\sum_{t \in T} \sum_{w \in W} \sum_{k \in K_{w}} b_{w k}^{t} D_{w k}^{t}\right)$. In the remaining cases in which there is not enough water to satisfy all the demands the relevance of the bilevel model and the importance of prices is made clear. As can be expected, the more water available ( $\beta$ is larger), the more water users have their demand satisfied.

In what follows, we consider the values of $\beta$ for which it is not possible to satisfy the total demand without charging prices, and pay attention to the satisfaction of water users measured by the socalled satisfaction index defined as the quotient between the total satisfaction given by the value of $f_{1}^{2}$ evaluated for the corresponding solution and the satisfaction of all the water users:

$$
I=\frac{f_{1}^{2}}{|T| \times|K|} .
$$


Table 6 summarizes the results for $T=12$. As mentioned above, this table does not include those values of $\beta$ for which this index is equal to 1 , that is, every water user receives as much water as he/she demands in every period. The first and the second columns of the table display the water system and the value of $\beta$. The third and fourth columns show the satisfaction index $I$ value of the optimal solution of the relaxed problem and the corresponding suboptimal solution, respectively. Finally, the remaining columns display the value of $I$ associated with the optimal solution of the bilevel problem when $\alpha$ is equal to $0,0.25,0.5$, and 0.75 . A symbol " $="$ is written when the index value coincides with the index value provided by the optimal solution of the relaxed problem. This value is an upper bound of the index value of every bilevel feasible solution. The results corresponding to $T=52$ and $T=365$ are shown in the Appendix. Since the results in all the tables are very similar (they are better for $T=365$ than for $T=52$, which is better than for $T=12$, but the differences are less than 0.02), we summarize the information for $T=12$.

Looking at Table 6 , as could be expected, the satisfaction index associated with every instance always increases as the available water is increased ( $\beta$ grows). The optimal solution of the relaxed problem provides the best values but, as mentioned above, in a hierarchical framework, the suboptimal solution is obtained and thus the satisfaction index actually computed is the one shown under suboptimal, which always provides the worst values. However, comparing the index value of the relaxed optimal solution with the indexes of the optimal solution of the bilevel problems, we can realize which value of $\alpha$ provides the best satisfaction and which value provides an index close enough to that. Note also that to obtain the optimal solution of the relaxed problem by means of a bilevel problem, in general it is needed to enforce $\alpha=0.75$, the largest value analyzed in this study. For large enough values of $\beta$, this also happens when $\alpha=0.5$ and $\alpha=0.25$ for some water systems.

\section{Conclusions}

In this paper we have proposed a decision tool based on bilevel optimization, which can be used by decision makers to be aware of the impact of different water-allocation policies that can involve charging fees when it is mandatory to take into account the decisions made at the next level of the decision-making process. For this purpose, we have proposed a multiobjective multifollower bilevel optimization model to manage water allocation in a hierarchical decentralized water system. The purpose is to guarantee environmental requirements as well as to make an efficient use of available water in terms of satisfying demand. Due to the hierarchical decision process involved, a bilevel model is proposed whose upper level decision maker, the central authority, aims first to satisfy minimum requirements. Second, he/she aims to maximize the overall satisfaction of the water user demands. In the process of deciding how to allocate the available water, the upper level is constrained by the behavior of the demand point managers who are at the lower level of the decision process. At this level, there are as many decision makers as demand points, each of them aiming to distribute the water assigned to them in accordance with the economic return. In addition, the central authority also has as a regulatory tool the possibility of setting fees to be paid by the demand point depending on how it decides to allocate the water. The model allows us to see the evolution of the users satisfaction as the value of prices charged and the water available vary. The model can be easily adapted to manage very different water systems as can be inferred from the very distinct water systems and drought scenarios dealt with in the computational experiments. 
The model resulting from this approach, a multiobjective lexicographic linear bilevel optimization problem, has been reformulated as a multiobjective lexicographic linear bilevel problem with one follower. Next, an exact procedure to solve the model has been proposed based on applying the KKT conditions of the lower level problem. Valid constants for the big- $M$ s involved in the linearization of the KKT conditions are derived.

The results of the extensive computational experiments carried out on a large set of benchmark instances, which considers different water systems, drought scenarios, and several planning periods, confirm the efficiency of the procedure since it is able to provide the optimal solution in reasonable computing times (very short in most cases). This avoids the need to develop heuristics or metaheuristics algorithms since commercial software can be used to solve the problems involved. Moreover, it allows us to foresee that this procedure will be key when dealing with stochastic inflows or demands in future research.

\section{Acknowledgments}

This research has been funded by the Spanish Ministry of Economy, Industry and Competitiveness under grant ECO2016-76567-C4-3-R, the Spanish Ministry of Science and Innovation under grant PID2019-104263RB-C43, and by the Gobierno de Aragón under grants E41-17R (FEDER 20142020 "Construyendo Europa desde Aragón") and E41-20R.

The authors gratefully acknowledge the anonymous referees whose comments have helped to improve the presentation of the paper.

\section{References}

Amouzegar, M., Moshirvaziri, K., 1999. Determining optimal pollution control policies: an application of bilevel programming. European Journal of Operational Research 119, 100-120.

Aviso, K., Tan, R., Culaba, A., Cruz, J. Jr, 2010. Bi-level fuzzy optimization approach for water exchange in eco-industrial parks. Process Safety and Environmental Protection 88, 31-40.

Babel, M., Gupta, A.D., Nayak, D., 2005. A model for optimal allocation of water to competing demands. Water Resources Management 19, 693-712.

Bard, J., 1998. Practical Bilevel Optimization. Algorithms and Applications. Kluwer Academic Publishers, Dordrecht.

Bi, R., Chen, C., Tang, J., Jia, X., Xiang, S., 2019. Two-level optimization model for water consumption based on water prices in eco-industrial parks. Resources, Conservation and Recycling 146, 308-315.

Bostian, M., Whittaker, G., Barnhart, B., Färe, R., Grosskopf, S., 2015. Valuing water quality tradeoffs at different spatial scales: an integrated approach using bilevel optimization. Water Resources and Economics 11, 1-12.

Brown, C., Lund, J., Cai, X., Reed, P., Zagona, E., Ostfeld, A., Hall, J., Characklis, G., Yu, W., Brekke, L., 2015. The future of water resources systems analysis: toward a scientific framework for sustainable water management. Water Resources Research 51, 6110-6124.

Calvete, H., Galé, C., 2007. Linear bilevel multi-follower programming with independent followers. Journal of Global Optimization 39, 3, 409-417.

Calvete, H., Galé, C., Dempe, S., Lohse, S., 2012. Bilevel problems over polyhedra with extreme point optimal solutions. Journal of Global Optimization 53, 3, 573-586.

Calvete, H., Mateo, P., 1995. An approach for the network flow problem with multiple objectives. Computers and Operations Research 22, 9, 971-983. 
Chalmardi, M., Camacho-Vallejo, J., 2019. A bi-level programming model for sustainable supply chain network design that considers incentives for using cleaner technologies. Journal of Cleaner Production 213, 10351050 .

Chen, Y., Lu, H., Li, J., Ren, L., He, L., 2017. A leader-follower-interactive method for regional water resources management with considering multiple water demands and eco-environmental constraints. Journal of Hydrology 548, 121-134.

Colson, B., Marcotte, P., Savard, G., 2007. An overview of bilevel programming. Annals of Operations Research 153, 235-256.

Dempe, S., 2002. Foundations of Bilevel Programming. Kluwer Academic Publishers, Dordrecht.

Dempe, S., 2018. Bilevel Optimization: Theory, Algorithms and Applications. Fakultät für Mathematik und Informatik, TU Bergakademie Freiberg, Freiberg. Preprint 2018-11.

Dempe, S., Zemkoho, A., 2020. Bilevel Optimization. Advances and Next Challenges. Springer, Berlin.

Ehrgott, M., 2005. Multicriteria Optimization (2nd edn). Springer, Berlin.

Guo, X., Hu, T., Zhang, T., Lv, Y., 2012. Bilevel model for multi-reservoir operating policy in inter-basin water transfersupply project. Journal of Hydrology 424 425, 252-263.

Hassing, J., Ipsen, N., Clausen, T., Larsen, H., Lindgaard-Jørgensen, P., 2009. Integrated Water Resources Management in Action. World Water Assessment Programme. Side Publications Series: Dialogue Paper, UNEP-DHI Centre for Water and Environment, Hørsholm, pp. 1-58.

Karsu, O., Erkan, H., 2020. Balance in resource allocation problems: a changing reference approach. OR Spectrum 42, 297-326.

Kleinert, T., Labbé, M., Plein, F., Schmidt, M., 2020. There's no free lunch: on the hardness of choosing a correct Big-M in bilevel optimization. Operations Research 68, 1716-1721.

Llopis-Albert, C., Merigó, J., Liao, H., Xu, Y., Grima-Olmedo, J., Grima-Olmedo, C., 2018. Water policies and conflict resolution of public participation decision-making processes using prioritized ordered weighted averaging (OWA) operators. Water Resources Management 32, 497-510.

Lv, Y., Huang, G., Li, Y., Yang, Z., Liu, Y., Cheng, G., 2010. Planning regional water resources system using an interval fuzzy bi-level programming method. Journal of Environmental Informatics 16, 43-56.

Mala-Jetmarova, H., Sultanova, N., Savic, D., 2017. Lost in optimisation of water distribution systems? A literature review of system operation. Environmental Modelling and Software 93, 209-254.

Önal, H., Darmawan, D., Johnson, S., 1995. A multilevel analysis of agricultural credit distribution in east java, indonesia. Computers and Operations Research 22, 2, 227-236.

Pineda, S., Morales, J., 2019. Solving linear bilevel problems using big-Ms: not all that glitters is gold. IEEE Transactions on Power Systems 34, 3, 2469-2471.

Roozbahani, R., Schreider, S., Abbasi, B., 2013. Economic sharing of basin water resources between competing stakeholders. Water Resources Management 27, 2965-2988.

Ruiz-Villaverde, A., García-Rubio, M., 2017. Public participation in European water management: from theory to practice. Water Resources Management 31, 2479-2495.

Sinha, A., Malo, P., Deb, K., 2018. A review on bilevel optimization: from classical to evolutionary approaches and applications. IEEE Transactions on Evolutionary Computation 22, 276-295.

Tayfur, G., 2017. Modern optimization methods in water resources planning, engineering and management. Water Resources Management 31, 10, 3205-3233.

Udías, A., Ríos-Insua, D., Cano, J., Fellag, H., 2012. Cost-efficient equitable water distribution in algeria: a bicriteria fair division problem with network constraints. International Transaction in Operational Research 19, 675693.

Xu, J., Tu, Y., Zeng, Z., 2012. Bilevel optimization of regional water resources allocation problem under fuzzy random environment. Journal of Water Resources Planning and Management 139, 246-264.

Zhao, L., Li, C., Huang, R., Si, S., Xue, J., Huang, W., Hu, Y., 2013. Harmonizing model with transfer tax on water pollution across regional boundaries in a China's lake basin. European Journal of Operational Research 225, 377382.

Zhu, X., Zhang, C., Fu, G., Li, Y., Ding, W., 2017. Bi-level optimization for determining operating strategies for interbasin water transfer-supply reservoirs. Water Resources Management 31, 4415-4432. 


\section{Appendix}

Tables A1-A6, respectively, display the computing times required for solving the MLMF-BP and the relaxed problem for the instances WS1-WS6. In each table, the first and second columns display, respectively, the value of the coefficients $\beta$ and $\alpha$. The remaining six columns display $t_{\text {bil }}$, the CPU time in seconds required to solve the MLMF-BP, and $t_{\text {rel }}$, the CPU time in seconds required by CPLEX to solve the relaxed problem depending on the periods of the planning horizon, respectively. The CPLEX stopping criterion was set at 7200 seconds. Only 5 of the 720 bilevel problems (all of them having $T=365$ ) were stopped before providing an optimal solution.

Table A1

Computing times of the instance WS1

\begin{tabular}{|c|c|c|c|c|c|c|c|}
\hline \multirow[b]{2}{*}{$\beta$} & \multirow[b]{2}{*}{$\alpha$} & \multicolumn{2}{|c|}{$T=12$} & \multicolumn{2}{|c|}{$T=52$} & \multicolumn{2}{|l|}{$T=365$} \\
\hline & & $t_{b i l}$ & $t_{r e l}$ & $t_{b i l}$ & $t_{r e l}$ & $t_{b i l}$ & $t_{r e l}$ \\
\hline \multirow[t]{4}{*}{0.1} & 0 & 1.07 & \multirow[t]{4}{*}{0.04} & 13.89 & \multirow[t]{4}{*}{0.11} & 180.94 & \multirow[t]{4}{*}{3.55} \\
\hline & 0.25 & 1.67 & & 20.22 & & 126.77 & \\
\hline & 0.5 & 0.49 & & 8.54 & & 153.42 & \\
\hline & 0.75 & 0.33 & & 1.82 & & 70.69 & \\
\hline \multirow[t]{4}{*}{0.2} & 0 & 0.96 & \multirow[t]{4}{*}{0.04} & 5.35 & \multirow[t]{4}{*}{0.10} & 7.75 & \multirow[t]{4}{*}{1.36} \\
\hline & 0.25 & 1.44 & & 34.63 & & 7.45 & \\
\hline & 0.5 & 0.90 & & 8.66 & & 7.59 & \\
\hline & 0.75 & 0.66 & & 3.24 & & 561.90 & \\
\hline \multirow[t]{4}{*}{0.3} & 0 & 0.89 & \multirow[t]{4}{*}{0.05} & 9.57 & \multirow[t]{4}{*}{0.11} & 2221.31 & \multirow[t]{4}{*}{1.35} \\
\hline & 0.25 & 1.47 & & 12.84 & & 1285.28 & \\
\hline & 0.5 & 0.85 & & 7.63 & & 96.73 & \\
\hline & 0.75 & 0.30 & & 2.09 & & 7.34 & \\
\hline \multirow[t]{4}{*}{0.4} & 0 & 0.60 & \multirow[t]{4}{*}{0.05} & 4.34 & \multirow[t]{4}{*}{0.11} & 7.23 & \multirow[t]{4}{*}{1.37} \\
\hline & 0.25 & 0.63 & & 5.04 & & 7.23 & \\
\hline & 0.5 & 0.68 & & 4.48 & & 115.26 & \\
\hline & 0.75 & 0.29 & & 1.84 & & 1057.37 & \\
\hline \multirow[t]{4}{*}{0.5} & 0 & 0.15 & \multirow[t]{4}{*}{0.06} & 0.58 & \multirow[t]{4}{*}{0.10} & 607.91 & \multirow[t]{4}{*}{0.94} \\
\hline & 0.25 & 0.13 & & 0.56 & & 62.66 & \\
\hline & 0.5 & 0.12 & & 0.50 & & 5.98 & \\
\hline & 0.75 & 0.11 & & 0.46 & & 5.91 & \\
\hline \multirow[t]{4}{*}{0.6} & 0 & 0.15 & \multirow[t]{4}{*}{0.05} & 0.59 & \multirow[t]{4}{*}{0.10} & 5.92 & \multirow[t]{4}{*}{0.93} \\
\hline & 0.25 & 0.13 & & 0.55 & & 31.06 & \\
\hline & 0.5 & 0.12 & & 0.49 & & 57.63 & \\
\hline & 0.75 & 0.11 & & 0.46 & & 160.39 & \\
\hline \multirow[t]{4}{*}{0.7} & 0 & 0.15 & 0.06 & 0.59 & 0.10 & 26.25 & 0.93 \\
\hline & 0.25 & 0.13 & & 0.55 & & 5.55 & \\
\hline & 0.5 & 0.12 & & 0.49 & & 5.44 & \\
\hline & 0.75 & 0.11 & & 0.46 & & 5.42 & \\
\hline
\end{tabular}


Table A2

Computing times of the instance WS2

\begin{tabular}{|c|c|c|c|c|c|c|c|}
\hline \multirow[b]{2}{*}{$\beta$} & \multirow[b]{2}{*}{$\alpha$} & \multicolumn{2}{|c|}{$T=12$} & \multicolumn{2}{|c|}{$T=52$} & \multicolumn{2}{|l|}{$T=365$} \\
\hline & & $t_{b i l}$ & $t_{r e l}$ & $t_{b i l}$ & $t_{r e l}$ & $t_{b i l}$ & $t_{r e l}$ \\
\hline \multirow[t]{4}{*}{0.1} & 0 & 3.32 & \multirow[t]{4}{*}{0.08} & 31.73 & \multirow[t]{4}{*}{0.20} & 559.17 & \multirow[t]{4}{*}{5.14} \\
\hline & 0.25 & 3.50 & & 25.26 & & 296.83 & \\
\hline & 0.5 & 1.91 & & 11.82 & & 582.40 & \\
\hline & 0.75 & 0.57 & & 6.36 & & 583.45 & \\
\hline \multirow[t]{4}{*}{0.2} & 0 & 3.27 & \multirow[t]{4}{*}{0.08} & 21.65 & \multirow[t]{4}{*}{0.22} & 409.48 & \multirow[t]{4}{*}{3.31} \\
\hline & 0.25 & 4.50 & & 28.48 & & 134.16 & \\
\hline & 0.5 & 2.28 & & 52.09 & & 16.43 & \\
\hline & 0.75 & 0.54 & & 6.91 & & 2081.76 & \\
\hline \multirow[t]{4}{*}{0.3} & 0 & 2.39 & \multirow[t]{4}{*}{0.08} & 25.87 & \multirow[t]{4}{*}{0.22} & 7200.66 & \multirow[t]{4}{*}{3.40} \\
\hline & 0.25 & 4.40 & & 36.27 & & 7200.65 & \\
\hline & 0.5 & 1.27 & & 49.46 & & 6849.79 & \\
\hline & 0.75 & 0.59 & & 9.97 & & 3747.03 & \\
\hline \multirow[t]{4}{*}{0.4} & 0 & 2.16 & \multirow[t]{4}{*}{0.08} & 27.83 & \multirow[t]{4}{*}{0.22} & 269.39 & \multirow[t]{4}{*}{3.38} \\
\hline & 0.25 & 3.75 & & 108.57 & & 15.83 & \\
\hline & 0.5 & 3.47 & & 15.50 & & 427.58 & \\
\hline & 0.75 & 0.54 & & 3.69 & & 6428.74 & \\
\hline \multirow[t]{4}{*}{0.5} & 0 & 1.99 & \multirow[t]{4}{*}{0.08} & 19.18 & \multirow[t]{4}{*}{0.21} & 3370.57 & \multirow[t]{4}{*}{3.40} \\
\hline & 0.25 & 3.26 & & 25.89 & & 3927.89 & \\
\hline & 0.5 & 1.43 & & 18.14 & & 373.39 & \\
\hline & 0.75 & 0.77 & & 3.64 & & 175.73 & \\
\hline \multirow[t]{4}{*}{0.6} & 0 & 1.33 & \multirow[t]{4}{*}{0.08} & 9.43 & \multirow[t]{4}{*}{0.22} & 13.52 & \multirow[t]{4}{*}{3.55} \\
\hline & 0.25 & 2.39 & & 10.31 & & 945.41 & \\
\hline & 0.5 & 1.57 & & 6.08 & & 183.59 & \\
\hline & 0.75 & 0.70 & & 2.86 & & 902.10 & \\
\hline \multirow[t]{4}{*}{0.7} & 0 & 0.28 & 0.07 & 1.29 & 0.18 & 1617.57 & 2.13 \\
\hline & 0.25 & 0.47 & & 1.34 & & 134.22 & \\
\hline & 0.5 & 0.49 & & 1.08 & & 111.55 & \\
\hline & 0.75 & 0.20 & & 0.99 & & 12.41 & \\
\hline
\end{tabular}

Table A3

Computing times of the instance WS3

\begin{tabular}{|c|c|c|c|c|c|c|c|}
\hline \multirow[b]{2}{*}{$\beta$} & \multirow[b]{2}{*}{$\alpha$} & \multicolumn{2}{|c|}{$T=12$} & \multicolumn{2}{|c|}{$T=52$} & \multicolumn{2}{|l|}{$T=365$} \\
\hline & & $t_{b i l}$ & $t_{\text {rel }}$ & $t_{b i l}$ & $t_{r e l}$ & $t_{b i l}$ & $t_{r e l}$ \\
\hline \multirow[t]{4}{*}{0.1} & 0 & 2.51 & \multirow[t]{4}{*}{0.04} & 11.68 & \multirow[t]{4}{*}{0.11} & 222.34 & \multirow[t]{4}{*}{3.41} \\
\hline & 0.25 & 3.40 & & 56.64 & & 36.52 & \\
\hline & 0.5 & 1.20 & & 8.01 & & 9.54 & \\
\hline & 0.75 & 0.95 & & 1.87 & & 10.25 & \\
\hline \multirow[t]{4}{*}{0.2} & 0 & 0.42 & \multirow[t]{4}{*}{0.04} & 2.88 & \multirow[t]{4}{*}{0.10} & 10.25 & \multirow[t]{4}{*}{1.95} \\
\hline & 0.25 & 0.71 & & 4.27 & & 10.25 & \\
\hline & 0.5 & 0.49 & & 4.64 & & 10.37 & \\
\hline & 0.75 & 0.47 & & 1.46 & & 5417.98 & \\
\hline
\end{tabular}

Continued

(C) 2021 The Authors. International Transactions in Operational Research (C) 2021 International Federation of Operational Research Societies 
Table A3

(Continued)

\begin{tabular}{|c|c|c|c|c|c|c|c|}
\hline \multirow[b]{2}{*}{$\beta$} & \multirow[b]{2}{*}{$\alpha$} & \multicolumn{2}{|c|}{$T=12$} & \multicolumn{2}{|c|}{$T=52$} & \multicolumn{2}{|l|}{$T=365$} \\
\hline & & $t_{b i l}$ & $t_{r e l}$ & $t_{b i l}$ & $t_{r e l}$ & $t_{b i l}$ & $t_{r e l}$ \\
\hline \multirow[t]{4}{*}{0.3} & 0 & 0.17 & \multirow[t]{4}{*}{0.04} & 0.70 & \multirow[t]{4}{*}{0.10} & 158.81 & \multirow[t]{4}{*}{3.85} \\
\hline & 0.25 & 0.19 & & 0.69 & & 11.06 & \\
\hline & 0.5 & 0.17 & & 0.61 & & 11.58 & \\
\hline & 0.75 & 0.16 & & 0.58 & & 11.55 & \\
\hline \multirow[t]{4}{*}{0.4} & 0 & 0.20 & \multirow[t]{4}{*}{0.04} & 0.73 & \multirow[t]{4}{*}{0.11} & 11.48 & \multirow[t]{4}{*}{3.12} \\
\hline & 0.25 & 0.19 & & 0.82 & & 11.48 & \\
\hline & 0.5 & 0.17 & & 0.73 & & 1213.20 & \\
\hline & 0.75 & 0.17 & & 0.67 & & 63.62 & \\
\hline \multirow[t]{4}{*}{0.5} & 0 & 0.19 & \multirow[t]{4}{*}{0.04} & 0.72 & \multirow[t]{4}{*}{0.10} & 10.02 & \multirow[t]{4}{*}{1.80} \\
\hline & 0.25 & 0.19 & & 0.80 & & 10.34 & \\
\hline & 0.5 & 0.17 & & 0.69 & & 9.87 & \\
\hline & 0.75 & 0.16 & & 0.61 & & 10.22 & \\
\hline \multirow[t]{4}{*}{0.6} & 0 & 0.59 & \multirow[t]{4}{*}{0.04} & 0.75 & \multirow[t]{4}{*}{0.11} & 9.80 & \multirow[t]{4}{*}{1.32} \\
\hline & 0.25 & 0.20 & & 0.83 & & 175.88 & \\
\hline & 0.5 & 0.17 & & 0.72 & & 27.68 & \\
\hline & 0.75 & 0.17 & & 0.61 & & 9.42 & \\
\hline \multirow[t]{4}{*}{0.7} & 0 & 0.18 & \multirow[t]{4}{*}{0.04} & 0.74 & \multirow[t]{4}{*}{0.11} & 9.82 & \multirow[t]{4}{*}{1.25} \\
\hline & 0.25 & 0.19 & & 0.93 & & 9.23 & \\
\hline & 0.5 & 0.18 & & 0.72 & & 9.12 & \\
\hline & 0.75 & 0.16 & & 0.61 & & 9.13 & \\
\hline
\end{tabular}

Table A4

Computing times of the instance WS4

\begin{tabular}{|c|c|c|c|c|c|c|c|}
\hline \multirow[b]{2}{*}{$\beta$} & \multirow[b]{2}{*}{$\alpha$} & \multicolumn{2}{|c|}{$T=12$} & \multicolumn{2}{|l|}{$T=52$} & \multicolumn{2}{|l|}{$T=365$} \\
\hline & & $t_{b i l}$ & $t_{r e l}$ & $t_{b i l}$ & $t_{r e l}$ & $t_{b i l}$ & $t_{\text {rel }}$ \\
\hline \multirow[t]{4}{*}{0.1} & 0 & 1.78 & \multirow[t]{4}{*}{0.07} & 20.61 & \multirow[t]{4}{*}{0.20} & 193.90 & \multirow[t]{4}{*}{6.74} \\
\hline & 0.25 & 8.82 & & 255.16 & & 323.54 & \\
\hline & 0.5 & 2.30 & & 46.31 & & 149.58 & \\
\hline & 0.75 & 0.81 & & 3.31 & & 13.61 & \\
\hline \multirow[t]{4}{*}{0.2} & 0 & 1.54 & \multirow[t]{4}{*}{0.08} & 12.88 & \multirow[t]{4}{*}{0.18} & 13.70 & \multirow[t]{4}{*}{2.88} \\
\hline & 0.25 & 2.84 & & 64.38 & & 13.76 & \\
\hline & 0.5 & 1.43 & & 17.50 & & 13.77 & \\
\hline & 0.75 & 0.48 & & 5.42 & & 7201.24 & \\
\hline \multirow[t]{4}{*}{0.3} & 0 & 1.12 & \multirow[t]{4}{*}{0.08} & 8.05 & \multirow[t]{4}{*}{0.20} & 5235.90 & \multirow[t]{4}{*}{2.74} \\
\hline & 0.25 & 1.90 & & 11.37 & & 534.20 & \\
\hline & 0.5 & 1.41 & & 7.92 & & 14.94 & \\
\hline & 0.75 & 0.45 & & 3.30 & & 14.99 & \\
\hline \multirow[t]{4}{*}{0.4} & 0 & 0.23 & \multirow[t]{4}{*}{0.07} & 1.00 & \multirow[t]{4}{*}{0.16} & 15.10 & \multirow[t]{4}{*}{1.76} \\
\hline & 0.25 & 0.26 & & 1.04 & & 15.07 & \\
\hline & 0.5 & 0.21 & & 1.00 & & 5064.09 & \\
\hline & 0.75 & 0.19 & & 0.93 & & 1615.83 & \\
\hline
\end{tabular}


Table A4

(Continued)

\begin{tabular}{|c|c|c|c|c|c|c|c|}
\hline \multirow[b]{2}{*}{$\beta$} & \multirow[b]{2}{*}{$\alpha$} & \multicolumn{2}{|c|}{$T=12$} & \multicolumn{2}{|c|}{$T=52$} & \multicolumn{2}{|c|}{$T=365$} \\
\hline & & $t_{b i l}$ & $t_{r e l}$ & $t_{b i l}$ & $t_{r e l}$ & $t_{b i l}$ & $t_{r e l}$ \\
\hline \multirow[t]{4}{*}{0.5} & 0 & 0.23 & \multirow[t]{4}{*}{0.07} & 1.01 & \multirow[t]{4}{*}{0.16} & 155.96 & \multirow[t]{4}{*}{1.75} \\
\hline & 0.25 & 0.26 & & 1.06 & & 12.60 & \\
\hline & 0.5 & 0.63 & & 0.88 & & 12.29 & \\
\hline & 0.75 & 0.18 & & 0.83 & & 12.27 & \\
\hline \multirow[t]{4}{*}{0.6} & 0 & 0.23 & \multirow[t]{4}{*}{0.07} & 1.01 & \multirow[t]{4}{*}{0.15} & 12.15 & \multirow[t]{4}{*}{1.72} \\
\hline & 0.25 & 0.26 & & 1.06 & & 652.18 & \\
\hline & 0.5 & 0.21 & & 0.89 & & 374.32 & \\
\hline & 0.75 & 0.18 & & 1.01 & & 73.87 & \\
\hline \multirow[t]{4}{*}{0.7} & 0 & 0.23 & \multirow[t]{4}{*}{0.07} & 1.01 & \multirow[t]{4}{*}{0.16} & 11.68 & \multirow[t]{4}{*}{1.68} \\
\hline & 0.25 & 0.26 & & 1.48 & & 11.27 & \\
\hline & 0.5 & 0.22 & & 0.89 & & 11.22 & \\
\hline & 0.75 & 0.18 & & 0.82 & & 11.14 & \\
\hline
\end{tabular}

Table A5

Computing times of the instance WS5

\begin{tabular}{|c|c|c|c|c|c|c|c|}
\hline \multirow[b]{2}{*}{$\beta$} & \multirow[b]{2}{*}{$\alpha$} & \multicolumn{2}{|c|}{$T=12$} & \multicolumn{2}{|c|}{$T=52$} & \multicolumn{2}{|l|}{$T=365$} \\
\hline & & $t_{b i l}$ & $t_{r e l}$ & $t_{b i l}$ & $t_{r e l}$ & $t_{b i l}$ & $t_{r e l}$ \\
\hline \multirow[t]{4}{*}{0.1} & 0 & 1.52 & 0.05 & 43.88 & 0.15 & 276.72 & 2.24 \\
\hline & 0.25 & 3.46 & & 105.33 & & 260.61 & \\
\hline & 0.5 & 1.40 & & 9.63 & & 271.90 & \\
\hline & 0.75 & 0.46 & & 2.35 & & 115.36 & \\
\hline \multirow[t]{4}{*}{0.2} & 0 & 1.48 & 0.07 & 16.85 & 0.14 & 10.42 & 2.03 \\
\hline & 0.25 & 2.71 & & 50.90 & & 10.47 & \\
\hline & 0.5 & 1.31 & & 14.84 & & 10.57 & \\
\hline & 0.75 & 0.41 & & 2.77 & & 4544.62 & \\
\hline \multirow[t]{4}{*}{0.3} & 0 & 1.40 & 0.07 & 10.19 & 0.14 & 3892.10 & 2.05 \\
\hline & 0.25 & 2.09 & & 24.26 & & 5908.68 & \\
\hline & 0.5 & 1.06 & & 10.43 & & 227.78 & \\
\hline & 0.75 & 0.35 & & 3.75 & & 11.88 & \\
\hline \multirow[t]{4}{*}{0.4} & 0 & 0.86 & 0.07 & 5.86 & 0.13 & 11.88 & 2.08 \\
\hline & 0.25 & 1.35 & & 9.05 & & 11.89 & \\
\hline & 0.5 & 0.70 & & 5.30 & & 2817.52 & \\
\hline & 0.75 & 0.38 & & 3.44 & & 4995.98 & \\
\hline \multirow[t]{4}{*}{0.5} & 0 & 0.19 & 0.07 & 0.74 & 0.12 & 754.21 & 1.36 \\
\hline & 0.25 & 0.20 & & 0.78 & & 121.10 & \\
\hline & 0.5 & 0.16 & & 0.81 & & 9.80 & \\
\hline & 0.75 & 0.15 & & 0.82 & & 9.64 & \\
\hline \multirow[t]{4}{*}{0.6} & 0 & 0.19 & 0.07 & 0.72 & 0.13 & 9.63 & 1.30 \\
\hline & 0.25 & 0.20 & & 0.78 & & 82.20 & \\
\hline & 0.5 & 0.17 & & 0.80 & & 438.61 & \\
\hline & 0.75 & 0.15 & & 0.73 & & 205.93 & \\
\hline
\end{tabular}

(C) 2021 The Authors. International Transactions in Operational Research (C) 2021 International Federation of Operational Research Societies 
Table A5

(Continued)

\begin{tabular}{|c|c|c|c|c|c|c|c|}
\hline \multirow[b]{2}{*}{$\beta$} & \multirow[b]{2}{*}{$\alpha$} & \multicolumn{2}{|c|}{$T=12$} & \multicolumn{2}{|c|}{$T=52$} & \multicolumn{2}{|c|}{$T=365$} \\
\hline & & $t_{b i l}$ & $t_{r e l}$ & $t_{b i l}$ & $t_{\text {rel }}$ & $t_{b i l}$ & $t_{r e l}$ \\
\hline \multirow[t]{4}{*}{0.7} & 0 & 0.19 & \multirow[t]{4}{*}{0.07} & 0.72 & \multirow[t]{4}{*}{0.12} & 45.22 & \multirow[t]{4}{*}{1.27} \\
\hline & 0.25 & 0.20 & & 0.78 & & 8.86 & \\
\hline & 0.5 & 0.17 & & 0.78 & & 8.84 & \\
\hline & 0.75 & 0.15 & & 0.80 & & 8.80 & \\
\hline
\end{tabular}

Table A6

Computing times of the instance WS6

\begin{tabular}{|c|c|c|c|c|c|c|c|}
\hline \multirow[b]{2}{*}{$\beta$} & \multirow[b]{2}{*}{$\alpha$} & \multicolumn{2}{|c|}{$T=12$} & \multicolumn{2}{|l|}{$T=52$} & \multicolumn{2}{|l|}{$T=365$} \\
\hline & & $t_{b i l}$ & $t_{r e l}$ & $t_{b i l}$ & $t_{r e l}$ & $t_{b i l}$ & $t_{r e l}$ \\
\hline \multirow[t]{4}{*}{0.1} & 0 & 3.34 & \multirow[t]{4}{*}{0.08} & 70.59 & \multirow[t]{4}{*}{0.24} & 485.46 & \multirow[t]{4}{*}{3.47} \\
\hline & 0.25 & 3.75 & & 99.56 & & 346.70 & \\
\hline & 0.5 & 1.50 & & 12.81 & & 629.89 & \\
\hline & 0.75 & 0.63 & & 3.15 & & 534.05 & \\
\hline \multirow[t]{4}{*}{0.2} & 0 & 1.30 & \multirow[t]{4}{*}{0.08} & 16.04 & \multirow[t]{4}{*}{0.23} & 242.78 & \multirow[t]{4}{*}{3.20} \\
\hline & 0.25 & 3.94 & & 24.02 & & 16.18 & \\
\hline & 0.5 & 1.51 & & 55.97 & & 16.03 & \\
\hline & 0.75 & 1.11 & & 4.90 & & 2264.29 & \\
\hline \multirow[t]{4}{*}{0.3} & 0 & 2.61 & \multirow[t]{4}{*}{0.08} & 19.23 & \multirow[t]{4}{*}{0.22} & 7200.61 & \multirow[t]{4}{*}{3.39} \\
\hline & 0.25 & 4.99 & & 244.81 & & 7200.62 & \\
\hline & 0.5 & 3.19 & & 65.83 & & 4041.26 & \\
\hline & 0.75 & 0.64 & & 3.72 & & 357.03 & \\
\hline \multirow[t]{4}{*}{0.4} & 0 & 2.26 & \multirow[t]{4}{*}{0.08} & 15.29 & \multirow[t]{4}{*}{0.22} & 15.77 & \multirow[t]{4}{*}{3.33} \\
\hline & 0.25 & 2.86 & & 69.67 & & 15.62 & \\
\hline & 0.5 & 1.82 & & 10.72 & & 1938.42 & \\
\hline & 0.75 & 0.79 & & 3.88 & & 5105.76 & \\
\hline \multirow[t]{4}{*}{0.5} & 0 & 1.33 & \multirow[t]{4}{*}{0.08} & 19.99 & \multirow[t]{4}{*}{0.22} & 3743.76 & \multirow[t]{4}{*}{3.23} \\
\hline & 0.25 & 2.11 & & 13.90 & & 1225.49 & \\
\hline & 0.5 & 2.26 & & 8.38 & & 259.25 & \\
\hline & 0.75 & 0.49 & & 2.53 & & 13.30 & \\
\hline \multirow[t]{4}{*}{0.6} & 0 & 0.25 & \multirow[t]{4}{*}{0.08} & 1.27 & \multirow[t]{4}{*}{0.19} & 13.27 & \multirow[t]{4}{*}{1.97} \\
\hline & 0.25 & 0.24 & & 1.34 & & 357.14 & \\
\hline & 0.5 & 0.22 & & 0.95 & & 94.36 & \\
\hline & 0.75 & 0.19 & & 0.89 & & 395.56 & \\
\hline \multirow[t]{4}{*}{0.7} & 0 & 0.66 & 0.08 & 1.26 & 0.19 & 743.10 & 1.93 \\
\hline & 0.25 & 0.24 & & 1.33 & & 428.40 & \\
\hline & 0.5 & 0.60 & & 0.95 & & 12.14 & \\
\hline & 0.75 & 0.19 & & 0.93 & & 12.05 & \\
\hline
\end{tabular}

(C) 2021 The Authors.

International Transactions in Operational Research (C) 2021 International Federation of Operational Research Societies 
Tables A7 and A8 display the satisfaction index for $T=12$ and $T=365$, respectively. They have the same structure as Table 6 .

Table A7

The satisfaction index $I$ for $T=52$

\begin{tabular}{|c|c|c|c|c|c|c|c|}
\hline & & Relaxed & & Bilevel & & & \\
\hline & $\beta$ & Optimal & Suboptimal & $\alpha=0$ & $\alpha=0.25$ & $\alpha=0.5$ & $\alpha=0.75$ \\
\hline WS1 & 0.1 & 0.42 & 0.19 & 0.28 & 0.35 & $=$ & $=$ \\
\hline & 0.2 & 0.70 & 0.43 & 0.53 & 0.60 & 0.67 & $=$ \\
\hline & 0.3 & 0.85 & 0.69 & 0.75 & 0.80 & 0.83 & $=$ \\
\hline & 0.4 & 0.94 & 0.87 & 0.92 & 0.93 & $=$ & $=$ \\
\hline WS2 & 0.1 & 0.34 & 0.16 & 0.22 & 0.30 & 0.33 & $=$ \\
\hline & 0.2 & 0.60 & 0.32 & 0.41 & 0.49 & 0.56 & $=$ \\
\hline & 0.3 & 0.74 & 0.49 & 0.58 & 0.64 & 0.70 & $=$ \\
\hline & 0.4 & 0.84 & 0.68 & 0.73 & 0.78 & 0.82 & $=$ \\
\hline & 0.5 & 0.91 & 0.82 & 0.87 & 0.89 & $=$ & $=$ \\
\hline & 0.6 & 0.97 & 0.94 & 0.96 & $=$ & $=$ & $=$ \\
\hline WS3 & 0.1 & 0.72 & 0.50 & 0.58 & 0.64 & 0.70 & $=$ \\
\hline & 0.2 & 0.95 & 0.88 & 0.92 & 0.93 & $=$ & $=$ \\
\hline WS4 & 0.1 & 0.60 & 0.33 & 0.42 & 0.50 & 0.57 & $=$ \\
\hline & 0.2 & 0.84 & 0.68 & 0.74 & 0.79 & 0.83 & $=$ \\
\hline & 0.3 & 0.96 & 0.92 & 0.95 & $=$ & $=$ & $=$ \\
\hline WS5 & 0.1 & 0.45 & 0.20 & 0.27 & 0.36 & 0.43 & $=$ \\
\hline & 0.2 & 0.72 & 0.47 & 0.55 & 0.62 & 0.69 & $=$ \\
\hline & 0.3 & 0.87 & 0.73 & 0.79 & 0.82 & 0.86 & $=$ \\
\hline & 0.4 & 0.96 & 0.91 & 0.95 & $=$ & $=$ & $=$ \\
\hline WS6 & 0.1 & 0.36 & 0.16 & 0.23 & 0.31 & 0.35 & $=$ \\
\hline & 0.2 & 0.63 & 0.35 & 0.44 & 0.52 & 0.59 & $=$ \\
\hline & 0.3 & 0.78 & 0.57 & 0.64 & 0.70 & 0.75 & $=$ \\
\hline & 0.4 & 0.88 & 0.76 & 0.81 & 0.84 & 0.87 & $=$ \\
\hline & 0.5 & 0.95 & 0.90 & 0.93 & $=$ & $=$ & $=$ \\
\hline
\end{tabular}

Table A8

The satisfaction index $I$ for $T=365$

\begin{tabular}{|c|c|c|c|c|c|c|c|}
\hline & \multirow[b]{2}{*}{$\beta$} & \multicolumn{2}{|l|}{ Relaxed } & \multicolumn{4}{|c|}{ Bilevel } \\
\hline & & Optimal & Suboptimal & $\alpha=0$ & $\alpha=0.25$ & $\alpha=0.5$ & $\alpha=0.75$ \\
\hline \multirow[t]{4}{*}{ WS1 } & 0.1 & 0.43 & 0.20 & 0.28 & 0.36 & $=$ & $=$ \\
\hline & 0.2 & 0.71 & 0.43 & 0.54 & 0.61 & 0.68 & $=$ \\
\hline & 0.3 & 0.85 & 0.69 & 0.76 & 0.80 & 0.84 & $=$ \\
\hline & 0.4 & 0.94 & 0.87 & 0.92 & $=$ & $=$ & $=$ \\
\hline \multirow[t]{2}{*}{ WS2 } & 0.1 & 0.35 & 0.16 & 0.22 & 0.30 & 0.34 & $=$ \\
\hline & 0.2 & 0.61 & 0.32 & 0.41 & 0.50 & 0.58 & $=$ \\
\hline
\end{tabular}

Continued 
Table A8

(Continued)

\begin{tabular}{|c|c|c|c|c|c|c|c|}
\hline & \multirow[b]{2}{*}{$\beta$} & \multicolumn{2}{|l|}{ Relaxed } & \multicolumn{4}{|l|}{ Bilevel } \\
\hline & & Optimal & Suboptimal & $\alpha=0$ & $\alpha=0.25$ & $\alpha=0.5$ & $\alpha=0.75$ \\
\hline & 0.3 & 0.74 & 0.49 & 0.58 & 0.65 & 0.72 & $=$ \\
\hline & 0.4 & 0.84 & 0.68 & 0.74 & 0.79 & 0.82 & $=$ \\
\hline & 0.5 & 0.92 & 0.82 & 0.87 & 0.90 & 0.91 & $=$ \\
\hline & 0.6 & 0.97 & 0.94 & $=$ & $=$ & $=$ & $=$ \\
\hline \multirow[t]{2}{*}{ WS3 } & 0.1 & 0.73 & 0.50 & 0.59 & 0.65 & 0.71 & $=$ \\
\hline & 0.2 & 0.95 & 0.89 & 0.92 & 0.94 & $=$ & $=$ \\
\hline \multirow[t]{3}{*}{ WS4 } & 0.1 & 0.61 & 0.33 & 0.42 & 0.51 & 0.58 & $=$ \\
\hline & 0.2 & 0.85 & 0.68 & 0.75 & 0.79 & 0.83 & $=$ \\
\hline & 0.3 & 0.96 & 0.92 & 0.95 & $=$ & $=$ & $=$ \\
\hline \multirow[t]{4}{*}{ WS5 } & 0.1 & 0.45 & 0.20 & 0.27 & 0.36 & 0.43 & $=$ \\
\hline & 0.2 & 0.73 & 0.47 & 0.56 & 0.63 & 0.70 & $=$ \\
\hline & 0.3 & 0.88 & 0.73 & 0.79 & 0.83 & 0.86 & $=$ \\
\hline & 0.4 & 0.96 & 0.91 & 0.95 & $=$ & $=$ & $=$ \\
\hline \multirow[t]{5}{*}{ WS6 } & 0.1 & 0.37 & 0.17 & 0.23 & 0.31 & 0.36 & $=$ \\
\hline & 0.2 & 0.65 & 0.36 & 0.45 & 0.54 & 0.61 & $=$ \\
\hline & 0.3 & 0.78 & 0.57 & 0.65 & 0.71 & 0.76 & $=$ \\
\hline & 0.4 & 0.88 & 0.76 & 0.81 & 0.85 & 0.87 & $=$ \\
\hline & 0.5 & 0.95 & 0.90 & 0.94 & $=$ & $=$ & $=$ \\
\hline
\end{tabular}

